\title{
Lattice Boltzmann simulations of thermal flows beyond the Boussinesq and ideal-gas approximations
}

\author{
Rongzong Huang $\odot,{ }^{1, *}$ Lijuan Lan, ${ }^{2}$ and Qing $\mathrm{Li}^{1, \dagger}$ \\ ${ }^{1}$ School of Energy Science and Engineering, Central South University, 410083 Changsha, China \\ ${ }^{2}$ School of Automation, Central South University, 410083 Changsha, China
}

(Received 22 June 2020; accepted 9 September 2020; published 7 October 2020)

\begin{abstract}
In this work, the recent lattice Boltzmann model with self-tuning equation of state (EOS) [R. Huang et al., J. Comput. Phys. 392, 227 (2019)] is improved in three aspects to simulate the thermal flows beyond the Boussinesq and ideal-gas approximations. First, an improved scheme is proposed to eliminate the additional cubic terms of velocity, which can significantly improve the numerical accuracy. Second, a local scheme is proposed to calculate the density gradient instead of the conventional finite-difference scheme. Third, a scaling factor is introduced into the lattice sound speed, which can be adjusted to effectively enhance numerical stability. The thermal Couette flow of a nonattracting rigid-sphere fluid, which is described by the Carnahan-Starling EOS, is first simulated, and the better performance of the present improvements on the numerical accuracy and stability is demonstrated. As a further application, the turbulent Rayleigh-Bénard convection in a supercritical fluid slightly above its critical point, which is described by the van der Waals EOS, is successfully simulated by the present lattice Boltzmann model. The piston effect of the supercritical fluid is successfully captured, which induces a fast and homogeneous increase of the temperature in the bulk region, and the time evolution from the initiation of heating to the final turbulent state is analyzed in detail and divided into five stages.
\end{abstract}

DOI: 10.1103/PhysRevE.102.043304

\section{INTRODUCTION}

The lattice Boltzmann (LB) method, developed over the past three decades, has been exploited to simulate various flow problems, including the fluid-structure interactions [1,2], porous media flows [3,4], multiphase flows [5-7], phase change problems [8-10], etc. In this method, the flow process is depicted by the tempospatial evolution of the distribution function (DF), which is governed by the mesoscopic LB equation, while the concerned macroscopic quantities, like the density, velocity, and temperature, and even the viscous stress and heat flux, can be easily and locally obtained from the knowledge of the DF. The thermal flow problems have been widely simulated by the LB method since the early stage of its development [11], and the corresponding thermal LB model has evolved into a fundamental starting point for the further applications of the LB method to many challenging problems involving thermal effects. Generally, the existing thermal LB models can be grouped into the multispeed approach [12-14], double-distribution-function (DDF) approach [15-17], and hybrid approach [18-20], among which the DDF

\footnotetext{
*rongzong.huang@csu.edu.cn

†Corresponding author: qingli@csu.edu.cn
}

Published by the American Physical Society under the terms of the Creative Commons Attribution 4.0 International license. Further distribution of this work must maintain attribution to the author(s) and the published article's title, journal citation, and DOI. approach is most widely employed due to its simplicity and flexibility. In the DDF approach, double DFs are adopted with one for the velocity field (i.e., the mass-momentum conservation equations) and the other for the temperature field (i.e., the energy conservation equation), and both the two involved LB equations can be constructed based on the standard lattice.

With the approximation that the thermal energy can be viewed as a scalar passively advected by the fluid flow, several passive-scalar thermal LB models [21-23] are proposed, where the viscous dissipation and compression work are not considered. Starting with the Boltzmann equation in kinetic theory, some DDF LB models [24-26] are developed in an $a$ priori manner for the simulation of thermal flows, in which the viscous dissipation and compression work can be naturally incorporated. In these thermal LB models [21-26], the recovered equation of state (EOS) is decoupled from the temperature and can be generally written as $p=\rho c_{s}^{2}$ with $c_{s}$ denoting the constant lattice sound speed. Consequently, these models are only applicable to the thermal flows under the Boussinesq approximation. Based on the DDF kinetic model constructed by Guo et al. [25], Li et al. [27] and Feng et al. [28] successfully developed two- and three-dimensional coupling LB models, respectively, which can recover the ideal-gas EOS just like the kinetic model does. A correction term is introduced to compensate for the cubic defect of the standard lattice $[27,28]$, as previously analyzed by Prasianakis and Karlin [29]. To enhance the numerical stability, Fei and Luo [30] employed the cascaded collision operator in the LB equation for velocity field. Recently, Saadat et al. [16] 
constant lattice sound speed $c_{s}$

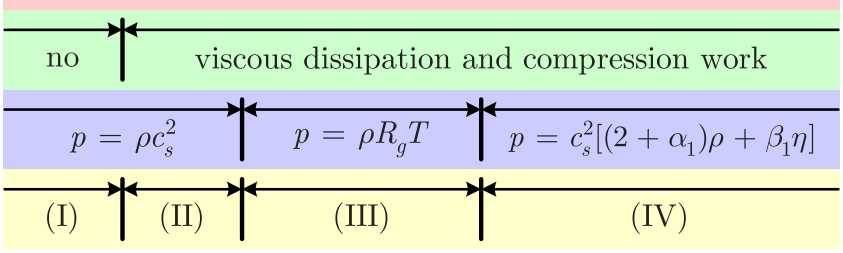

FIG. 1. Classification of the DDF LB models for thermal flows based on the consideration of viscous dissipation and compression work and the recovered EOS.

presented a DDF LB model for compressible flows, where the correction terms are designed under the acoustic scaling rather than the common diffusive scaling. In these thermal LB models [16,27,28,30], the recovered EOS is exactly the ideal-gas EOS $p=\rho R_{g} T$, implying that these models can handle the thermal flows of ideal gas with a large temperature difference when the Boussinesq approximation is invalid. Most recently, Huang et al. [17] developed a novel DDF LB model with self-tuning EOS for simulating coupled thermohydrodynamic flows. The recovered EOS is given as $p=$ $c_{s}^{2}\left[\left(2+\alpha_{1}\right) \rho+\beta_{1} \eta\right]\left(\alpha_{1}\right.$ and $\beta_{1}$ are the constant coefficients), which can be self-tuned via the built-in variable $\eta$ once the EOS for fluid is directly specified in practice. Thus, Huang et al.'s model is also not limited by the Boussinesq approximation and can even handle the thermal flows of nonideal gases.

From the above introduction, it can be seen that the existing DDF LB models for thermal flows can be further divided into four classes based on the consideration of viscous dissipation and compression work and the recovered EOS, as shown by Fig. 1. In class (I) models [21-23], the viscous dissipation and compression work are not considered, and the recovered EOS is the decoupling EOS $p=\rho c_{s}^{2}$. In class (II) models [24-26], the viscous dissipation and compression work can be naturally incorporated, and the recovered EOS remains the decoupling EOS. Therefore, both the class (I) and class (II) models are limited to the thermal flows under the Boussinesq approximation. The class (III) models $[16,27,28,30]$ can recover the ideal-gas EOS $p=\rho R_{g} T$, and thus the Boussinesq approximation can be relaxed. However, these class models are only applicable to the thermal flows of ideal gas. The class (IV) model [17] recovers a self-tuning EOS $p=c_{s}^{2}[(2+$ $\left.\alpha_{1}\right) \rho+\beta_{1} \eta$ ], which can be arbitrarily specified in practice. As a result, this model can handle the general thermal flows beyond both the Boussinesq and ideal-gas approximations. In this work, the class (IV) model, i.e., the recent LB model with self-tuning EOS [17], is focused on and is improved in three aspects, which can help to achieve better accuracy, localizability, and stability. To be specific, an improved scheme is proposed to eliminate the additional cubic terms of velocity, a local scheme is proposed to calculate the density gradient, and a scaling factor is introduced into the lattice sound speed. The remainder of this work is organized as follows. In Sec. II, the DDF LB model with self-tuning EOS for thermal flows is briefly introduced, and in Sec. III, the improvements in three aspects are presented in detail. Numerical validations are performed in Sec. IV, and a brief conclusion is drawn in Sec. V.

\section{LB MODEL WITH SELF-TUNING EOS}

In this section, the recent LB model with self-tuning EOS for thermal flows is briefly introduced for the sake of completeness, which is based on the DDF framework and the standard lattice. The density $\mathrm{DF} f_{i}(\mathbf{x}, t)$ is adopted for the mass-momentum conservation equations, and the total energy DF $g_{i}(\mathbf{x}, t)$ is adopted for the energy conservation equation. The two-dimensional standard lattice (i.e., the D2Q9 lattice) is considered here for simplicity, and the extension to the three-dimensional standard lattice is straightforward though tedious. The nine discrete velocities are given as [31]

$$
\mathbf{e}_{i}= \begin{cases}c(0,0)^{\mathrm{T}}, & i=0, \\ c(\cos [(i-1) \pi / 2], \sin [(i-1) \pi / 2])^{\mathrm{T}}, & i=1,2,3,4 \\ \sqrt{2} c(\cos [(2 i-1) \pi / 4], \sin [(2 i-1) \pi / 4])^{\mathrm{T}}, & i=5,6,7,8\end{cases}
$$

where $c=\delta_{x} / \delta_{t}$ is the lattice speed, $\delta_{x}$ is the lattice spacing, and $\delta_{t}$ is the time step.

\section{A. LB equation for density DF}

The LB equation with multiple-relaxation-time (MRT) collision operator for the density DF can be written as [17]

$$
\begin{aligned}
& f_{i}\left(\mathbf{x}+\mathbf{e}_{i} \delta_{t}, t+\delta_{t}\right)=\bar{f}_{i}(\mathbf{x}, t), \\
\overline{\mathbf{m}}= & \mathbf{m}+\delta_{t} \mathbf{F}_{m}-\mathbf{S}\left(\mathbf{m}-\mathbf{m}^{\mathrm{eq}}+\frac{\delta_{t}}{2} \mathbf{F}_{m}\right) \\
& -\mathbf{R}\left(\mathbf{I}-\frac{\mathbf{S}}{2}\right)\left(\mathbf{m}-\mathbf{m}^{\mathrm{eq}}+\frac{\delta_{t}}{2} \mathbf{F}_{m}\right) \\
& -\delta_{x} \mathbf{T} \cdot \nabla \rho-\delta_{x} \mathbf{X} \cdot \nabla \eta,
\end{aligned}
$$

where Eq. (2a) denotes the linear streaming process in velocity space, and Eq. (2b) denotes the local collision process in moment space. Note that the dependence of Eq. (2b) on $\mathbf{x}$ and $t$ is not shown to abbreviate the notation. As compared with the original work by Huang et al. [17], $\nabla \rho$ and $\nabla \eta$ are involved in the present collision process instead of $\nabla \rho$ and $\nabla p_{\mathrm{LBE}}$, and the calculation of $\nabla \rho$ and $\nabla \eta$ will be discussed in Sec. III B. Here, $p_{\text {LBE }}$ refers to the EOS recovered by the LB equation. The moment of density DF $\mathbf{m}$ in Eq. (2b) is obtained via the space transformation $\mathbf{m}=\mathbf{M}\left(f_{i}\right)^{\mathrm{T}}$, and the post-collision density $\mathrm{DF} \bar{f}_{i}$ in Eq. (2a) is obtained via the inverse transformation $\left(\bar{f}_{i}\right)^{\mathrm{T}}=\mathbf{M}^{-1} \overline{\mathbf{m}}$, with $\mathbf{M}$ denoting the 
dimensionless orthogonal transformation matrix [32,33]

$$
\mathbf{M}=\left[\begin{array}{ccccccccc}
1 & 1 & 1 & 1 & 1 & 1 & 1 & 1 & 1 \\
-4 & -1 & -1 & -1 & -1 & 2 & 2 & 2 & 2 \\
4 & -2 & -2 & -2 & -2 & 1 & 1 & 1 & 1 \\
0 & 1 & 0 & -1 & 0 & 1 & -1 & -1 & 1 \\
0 & -2 & 0 & 2 & 0 & 1 & -1 & -1 & 1 \\
0 & 0 & 1 & 0 & -1 & 1 & 1 & -1 & -1 \\
0 & 0 & -2 & 0 & 2 & 1 & 1 & -1 & -1 \\
0 & 1 & -1 & 1 & -1 & 0 & 0 & 0 & 0 \\
0 & 0 & 0 & 0 & 0 & 1 & -1 & 1 & -1
\end{array}\right] .
$$

The macroscopic density $\rho$ and momentum $\rho \mathbf{u}$ are defined as

$$
\rho=\sum_{i} f_{i}, \quad \rho \mathbf{u}=\sum_{i} \mathbf{e}_{i} f_{i}+\frac{\delta_{t}}{2} \mathbf{F},
$$

where $\mathbf{F}$ is an external force like gravity.
In the LB equation for density DF, the equilibrium moment function $\mathbf{m}^{\text {eq }}$ is given as $[17,34]$

$$
\begin{aligned}
\mathbf{m}^{\mathrm{eq}}= & {\left[\rho, 2 \alpha_{1} \rho+2 \beta_{1} \eta+3 \rho|\hat{\mathbf{u}}|^{2}, \alpha_{2} \rho+\beta_{2} \eta-3 \rho|\hat{\mathbf{u}}|^{2}+9 \rho \hat{u}_{x}^{2} \hat{u}_{y}^{2},\right.} \\
& \rho \hat{u}_{x},-\rho \hat{u}_{x}+3 \rho \hat{u}_{x} \hat{u}_{y}^{2}, \rho \hat{u}_{y},-\rho \hat{u}_{y}+3 \rho \hat{u}_{y} \hat{u}_{x}^{2}, \\
& \left.\rho \hat{u}_{x}^{2}-\rho \hat{u}_{y}^{2}, \rho \hat{u}_{x} \hat{u}_{y}\right]^{\mathrm{T}},
\end{aligned}
$$

where $\hat{\mathbf{u}} \equiv \mathbf{u} / c$, and $\eta$ is introduced to achieve the self-tuning EOS. The discrete force term in moment space $\mathbf{F}_{m}$ is given as [34]

$$
\begin{aligned}
\mathbf{F}_{m}= & {\left[0,6 \hat{\mathbf{F}} \cdot \hat{\mathbf{u}},-6 \hat{\mathbf{F}} \cdot \hat{\mathbf{u}}+9 \llbracket \hat{\mathbf{F}} \hat{\mathbf{u}} \mathbf{u} \hat{\mathbf{u}} \rrbracket_{x x y y}, \hat{F}_{x},\right.} \\
& -\hat{F}_{x}+3 \llbracket \hat{\mathbf{F}} \hat{\mathbf{u}} \rrbracket_{x y y}, \hat{F}_{y},-\hat{F}_{y}+3 \llbracket \hat{\mathbf{F}} \hat{\mathbf{u}} \mathbf{u} \rrbracket_{x x y}, \\
& \left.2 \hat{F}_{x} \hat{u}_{x}-2 \hat{F}_{y} \hat{u}_{y}, \hat{F}_{x} \hat{u}_{y}+\hat{F}_{y} \hat{u}_{x}\right]^{\mathrm{T}},
\end{aligned}
$$

where $\hat{\mathbf{F}} \equiv \mathbf{F} / c$, and the square brackets " $\llbracket . . . \rrbracket "$ and their subscript denote permutation and tensor index, respectively. For example, $\llbracket \mathbf{a b c} \rrbracket_{x y z}=a_{x} b_{y} c_{z}+b_{x} c_{y} a_{z}+c_{x} a_{y} b_{z}$. The collision matrix in moment space $\mathbf{S}$ is given as [34]

$$
\mathbf{S}=\left[\begin{array}{ccccccccc}
s_{0} & 0 & 0 & 0 & 0 & 0 & 0 & 0 & 0 \\
0 & s_{e} & k s_{\varepsilon} \omega_{e} & 0 & h \hat{u}_{x} s_{q} \omega_{e} & 0 & h \hat{u}_{y} s_{q} \omega_{e} & 0 & 0 \\
0 & 0 & s_{\varepsilon} & 0 & 0 & 0 & 0 & 0 & 0 \\
0 & 0 & 0 & s_{j} & 0 & 0 & 0 & 0 & 0 \\
0 & 0 & 0 & 0 & s_{q} & 0 & 0 & 0 & 0 \\
0 & 0 & 0 & 0 & 0 & s_{j} & 0 & 0 & 0 \\
0 & 0 & 0 & 0 & 0 & 0 & s_{q} & 0 & 0 \\
0 & 0 & 0 & 0 & 2 b \hat{u}_{x} s_{q} \omega_{p} & 0 & -2 b \hat{u}_{y} s_{q} \omega_{p} & s_{p} & 0 \\
0 & 0 & 0 & 0 & b \hat{u}_{y} s_{q} \omega_{p} & 0 & b \hat{u}_{x} s_{q} \omega_{p} & 0 & s_{p}
\end{array}\right],
$$

where $\omega_{e} \equiv s_{e} / 2-1$ and $\omega_{p} \equiv s_{p} / 2-1$. To correctly recover the macroscopic mass-momentum conservation equations, the coefficients $\alpha_{1}, \alpha_{2}, \beta_{1}$, and $\beta_{2}$ in $\mathbf{m}^{\text {eq }}$ and the coefficients $k, h$, and $b$ in $\mathbf{S}$ should satisfy [17]

$$
\alpha_{2}=-\frac{2 \alpha_{1}+\varpi+1}{1-\varpi}, \quad \beta_{2}=-\frac{2 \beta_{1}}{1-\varpi}, \quad k=1-\varpi, \quad h=\frac{6 \varpi(1-\varpi)}{1-3 \varpi}, \quad b=\frac{1-\varpi}{1-3 \varpi} .
$$

Here, $\alpha_{1}=-1$ and $\beta_{1}=1$ can be set as usual, and $\varpi$ is related to the bulk viscosity. To eliminate the additional cubic terms of velocity in recovering the viscous stress tensor, the correction terms $-\mathbf{R}\left(\mathbf{I}-\frac{\mathbf{S}}{2}\right)\left(\mathbf{m}-\mathbf{m}^{\mathrm{eq}}+\frac{\delta_{t}}{2} \mathbf{F}_{m}\right)-\delta_{x} \mathbf{T} \cdot \nabla \rho-\delta_{x} \mathbf{X} \cdot \nabla \eta$ are introduced into Eq. (2b), and the correction matrices $\mathbf{R}, \mathbf{T}$, and $\mathbf{X}$ should be set in the following form:

$$
\begin{aligned}
\mathbf{R} & =\left[\begin{array}{ccccccccc}
0 & 0 & 0 & 0 & 0 & 0 & 0 & 0 & 0 \\
0 & R_{11} & 0 & 0 & 0 & 0 & 0 & R_{17} & R_{18} \\
0 & 0 & 0 & 0 & 0 & 0 & 0 & 0 & 0 \\
0 & 0 & 0 & 0 & 0 & 0 & 0 & 0 & 0 \\
0 & 0 & 0 & 0 & 0 & 0 & 0 & 0 & 0 \\
0 & 0 & 0 & 0 & 0 & 0 & 0 & 0 & 0 \\
0 & 0 & 0 & 0 & 0 & 0 & 0 & 0 & 0 \\
0 & R_{71} & 0 & 0 & 0 & 0 & 0 & R_{77} & R_{78} \\
0 & R_{81} & 0 & 0 & 0 & 0 & 0 & R_{87} & R_{88}
\end{array}\right], \\
\mathbf{T} & =\left[\begin{array}{lllllllll}
\mathbf{0} & \mathbf{T}_{1} & \mathbf{0} & \mathbf{0} & \mathbf{0} & \mathbf{0} & \mathbf{0} & \mathbf{T}_{7} & \mathbf{T}_{8}
\end{array}\right]^{\mathrm{T}}, \\
\mathbf{X} & =\left[\begin{array}{lllllllll}
\mathbf{0} & \mathbf{X}_{1} & \mathbf{0} & \mathbf{0} & \mathbf{0} & \mathbf{0} & \mathbf{0} & \mathbf{X}_{7} & \mathbf{X}_{8}
\end{array}\right]^{\mathrm{T}},
\end{aligned}
$$

where the nonzero elements will be discussed in Sec. III A. 
Through the Chapman-Enskog analysis (see Ref. [34] for details), the macroscopic mass-momentum conservation equations can be recovered as

$$
\left\{\begin{array}{l}
\partial_{t} \rho+\nabla \cdot(\rho \mathbf{u})=0 \\
\partial_{t}(\rho \mathbf{u})+\nabla \cdot(\rho \mathbf{u u})=-\nabla p_{\mathrm{LBE}}+\mathbf{F}+\nabla \cdot \boldsymbol{\Pi}
\end{array}\right.
$$

where the $\operatorname{EOS} p_{\mathrm{LBE}}$ and viscous stress tensor $\Pi$ are

$$
\begin{aligned}
& p_{\mathrm{LBE}}=c_{s}^{2}\left[\left(2+\alpha_{1}\right) \rho+\beta_{1} \eta\right], \\
& \boldsymbol{\Pi}=\rho v\left[\nabla \mathbf{u}+(\nabla \mathbf{u})^{\mathrm{T}}-(\boldsymbol{\nabla} \cdot \mathbf{u}) \mathbf{I}\right]+\rho \varsigma(\boldsymbol{\nabla} \cdot \mathbf{u}) \mathbf{I} .
\end{aligned}
$$

Here, $c_{s}=c / \sqrt{3}$ is the lattice sound speed, $v=$ $c_{s}^{2} \delta_{t}\left(s_{p}^{-1}-0.5\right)$ is the kinematic viscosity, and $\varsigma=$ $\varpi c_{s}^{2} \delta_{t}\left(s_{e}^{-1}-0.5\right)$ is the bulk viscosity.

\section{B. LB equation for total energy DF}

The LB equation with MRT collision operator for the total energy DF can be written as [17]

$$
\begin{gathered}
g_{i}\left(\mathbf{x}+\mathbf{e}_{i} \delta_{t}, t+\delta_{t}\right)=\bar{g}_{i}(\mathbf{x}, t), \\
\overline{\mathbf{n}}=\mathbf{n}+\delta_{t} \mathbf{q}_{m}-\mathbf{L}\left(\mathbf{n}-\mathbf{n}^{\mathrm{eq}}+\frac{\delta_{t}}{2} \mathbf{q}_{m}\right)+c^{2} \mathbf{Y}\left(\frac{\mathbf{m}+\overline{\mathbf{m}}}{2}-\mathbf{m}^{\mathrm{eq}}\right),
\end{gathered}
$$

where Eqs. (12a) and (12b) denote the linear streaming process in velocity space and the local collision process in moment space, respectively, and the dependence of Eq. (12b) on $\mathbf{x}$ and $t$ is not shown to abbreviate the notation. Similar to Eq. (2), the moment of total energy DF $\mathbf{n}$ in Eq. (12b) is obtained via the transformation $\mathbf{n}=\mathbf{M}\left(g_{i}\right)^{\mathrm{T}}$, and the postcollision total energy DF $\bar{g}_{i}$ in Eq. (12a) is obtained via the inverse transformation $\left(\bar{g}_{i}\right)^{\mathrm{T}}=\mathbf{M}^{-1} \overline{\mathbf{n}}$. The macroscopic total energy $\rho e$ is defined as

$$
\rho e=\sum_{i} g_{i}+\frac{\delta_{t}}{2} q_{e},
$$

where $q_{e} \equiv \mathbf{u} \cdot \mathbf{F}+q$ is the equivalent source term, with $\mathbf{u} \cdot \mathbf{F}$ and $q$ representing the work done by force and the external source, respectively. In practical applications, once the density $\rho$, momentum $\rho \mathbf{u}$, and total energy $\rho e$ are defined by Eqs. (4) and (13), the other thermodynamic quantities, such as the internal energy $\rho \epsilon$ (defined as $\left.\rho \epsilon=\rho e-\rho|\mathbf{u}|^{2} / 2\right)$, the temperature $T$ (determined by $\rho$ and $\epsilon$ ), the pressure $p$ (given by the EOS for fluid), and the total enthalpy $\rho h$ (defined as $\rho h=\rho e+p$ ), can be easily and uniquely determined.

In the LB equation for total energy DF, the equilibrium moment function $\mathbf{n}^{\mathrm{eq}}$ is given as [17]

$$
\begin{aligned}
\mathbf{n}^{\mathrm{eq}}=[ & \rho e,-4\left(\rho e-\rho_{\mathrm{ref}} c_{\mathrm{ref}} T\right)+\gamma_{1} \rho_{\mathrm{ref}} c_{\mathrm{ref}} T, \\
& 4\left(\rho e-\rho_{\mathrm{ref}} c_{\mathrm{ref}} T\right)+\gamma_{2} \rho_{\mathrm{ref}} c_{\mathrm{ref}} T, \\
& \left.\rho h \hat{u}_{x},-\rho h \hat{u}_{x}, \rho h \hat{u}_{y},-\rho h \hat{u}_{y}, 0,0\right]^{\mathrm{T}},
\end{aligned}
$$

where $\rho_{\text {ref }}$ and $c_{\text {ref }}$ are the reference density and specific heat capacity, respectively, and the coefficients $\gamma_{1}$ and $\gamma_{2}$ are related to the heat conductivity. The discrete source term in moment space $\mathbf{q}_{m}$ is given as [17]

$$
\mathbf{q}_{m}=\left[q_{e}, \gamma_{1} q_{e}, \gamma_{2} q_{e}, q_{e} \hat{u}_{x},-q_{e} \hat{u}_{x}, q_{e} \hat{u}_{y},-q_{e} \hat{u}_{y}, 0,0\right]^{\mathrm{T}} .
$$

The collision matrix in moment space $\mathbf{L}$ is given as [35]

$$
\mathbf{L}=\left[\begin{array}{ccccccccc}
\sigma_{0} & 0 & 0 & 0 & 0 & 0 & 0 & 0 & 0 \\
0 & \sigma_{e} & 0 & 0 & 0 & 0 & 0 & 0 & 0 \\
0 & 0 & \sigma_{\varepsilon} & 0 & 0 & 0 & 0 & 0 & 0 \\
0 & 0 & 0 & \sigma_{j} & \sigma_{q} \omega_{j} & 0 & 0 & 0 & 0 \\
0 & 0 & 0 & 0 & \sigma_{q} & 0 & 0 & 0 & 0 \\
0 & 0 & 0 & 0 & 0 & \sigma_{j} & \sigma_{q} \omega_{j} & 0 & 0 \\
0 & 0 & 0 & 0 & 0 & 0 & \sigma_{q} & 0 & 0 \\
0 & 0 & 0 & 0 & 0 & 0 & 0 & \sigma_{p} & 0 \\
0 & 0 & 0 & 0 & 0 & 0 & 0 & 0 & \sigma_{p}
\end{array}\right],
$$

where $\omega_{j} \equiv \sigma_{j} / 2-1$. To correctly incorporate the viscous dissipation, the density-DF-related term $c^{2} \mathbf{Y}\left(\frac{\mathbf{m}+\overline{\mathbf{m}}}{2}-\mathbf{m}^{\mathrm{eq}}\right)$ is introduced into Eq. (12b), and the matrix $\mathbf{Y}$ should be set as follows [17]:

$$
\mathbf{Y}=\left[\begin{array}{ccccccccc}
0 & 0 & 0 & 0 & 0 & 0 & 0 & 0 & 0 \\
0 & 0 & 0 & 0 & 0 & 0 & 0 & 0 & 0 \\
0 & 0 & 0 & 0 & 0 & 0 & 0 & 0 & 0 \\
0 & \hat{u}_{x} / 3 & 0 & 0 & 0 & 0 & 0 & \hat{u}_{x} & 2 \hat{u}_{y} \\
0 & -\hat{u}_{x} / 3 & 0 & 0 & 0 & 0 & 0 & -\hat{u}_{x} & -2 \hat{u}_{y} \\
0 & \hat{u}_{y} / 3 & 0 & 0 & 0 & 0 & 0 & -\hat{u}_{y} & 2 \hat{u}_{x} \\
0 & -\hat{u}_{y} / 3 & 0 & 0 & 0 & 0 & 0 & \hat{u}_{y} & -2 \hat{u}_{x} \\
0 & 0 & 0 & 0 & 0 & 0 & 0 & 0 & 0 \\
0 & 0 & 0 & 0 & 0 & 0 & 0 & 0 & 0
\end{array}\right] .
$$

Through the Chapman-Enskog analysis (see Ref. [17] for details), the macroscopic energy conservation equation can be recovered as

$$
\partial_{t}(\rho e)+\nabla \cdot(\rho h \mathbf{u})=\nabla \cdot(\lambda \nabla T+\mathbf{u} \cdot \boldsymbol{\Pi})+\mathbf{u} \cdot \mathbf{F}+q,
$$

where $\lambda=\left(2 / 3+\gamma_{1} / 2+\gamma_{2} / 3\right) \rho_{\mathrm{ref}} c_{\mathrm{ref}} c^{2} \delta_{t}\left(\sigma_{j}^{-1}-0.5\right)$ is the heat conductivity, and $\Pi$ is the viscous stress tensor given by Eq. (11).

In the present DDF LB model with self-tuning EOS for thermal flows, the full coupling of the thermohydrodynamic effects can be automatically achieved by the self-tuning EOS $p_{\text {LBE }}$ [see Eq. (10)] and the viscous dissipation and compression work $\boldsymbol{\nabla} \cdot(\mathbf{u} \cdot \boldsymbol{\Pi})-\boldsymbol{\nabla} \cdot(p \mathbf{u})$ [see Eq. (18) and note that $-\nabla \cdot(p \mathbf{u})$ is combined into the convection term on the lefthand side of Eq. (18)] once $p_{\mathrm{LBE}}=p$ is set and $p=p(\rho, T)$ is specified. Therefore, the present LB model can handle the thermal flows beyond the Boussinesq approximation. Moreover, the EOS for fluid $p(\rho, T)$ can be arbitrarily specified, and then the built-in variable $\eta$ is inversely determined by $\eta=\left[c_{s}^{-2} p_{\mathrm{LBE}}-\left(2+\alpha_{1}\right) \rho\right] / \beta_{1}$. Thus, the present model with self-tuning EOS is not limited to the thermal flows of ideal gas, and it significantly extends the applicability of the LB method for thermal flow problems. 


\section{IMPROVEMENTS}

In this section, the recent LB model with self-tuning EOS for thermal flows is improved in three aspects. To be specific, an improved scheme is proposed to eliminate the additional cubic terms of velocity, which helps to improve the numerical accuracy. Then, a local scheme is proposed to calculate the density gradient, which retains the advantage of the local collision process. Last, a scaling factor is introduced into the lattice sound speed, which can be adjusted to enhance numerical stability.

\section{A. Eliminating cubic terms of velocity}

In the original work by Huang et al. [17], the additional cubic terms of velocity in recovering the viscous stress tensor are eliminated under additional approximations that the correction matrices $\mathbf{R}, \mathbf{T}$, and $\mathbf{X}$ in Eq. (2b) are of order $\mathrm{Ma}_{\text {lattice }}^{2}$, $\mathrm{Ma}_{\text {lattice }}^{3}$, and $\mathrm{Ma}_{\text {lattice }}^{3}$, respectively. Here, $\mathrm{Ma}_{\text {lattice }}$ denotes the lattice Mach number, which is defined based on the lattice sound speed rather than the physical sound speed. In this section, an improved scheme will be proposed to eliminate the additional cubic terms of velocity without any further approximations. The determinations of the nonzero elements in the correction matrices are summarized in the following, and the detailed derivation is given in Appendix. The nonzero elements $R_{11}, R_{17}$, and $R_{18}$ in the matrix $\mathbf{R}$ are determined by

$$
\boldsymbol{\Psi}\left[\begin{array}{c}
\left(2-s_{e}\right) R_{11} \\
\left(2-s_{p}\right) R_{17} \\
\left(2-s_{p}\right) R_{18}
\end{array}\right]=2\left(2-s_{e}\right)\left[\begin{array}{l}
A_{11} \\
A_{17} \\
A_{18}
\end{array}\right],
$$

where $\boldsymbol{\Psi}$ is a $3 \times 3$ matrix

$$
\boldsymbol{\Psi}=\frac{2}{3}\left[\begin{array}{ccc}
6 \varpi s_{e}^{-1} & 0 & 0 \\
0 & 2 s_{p}^{-1} & 0 \\
0 & 0 & s_{p}^{-1}
\end{array}\right]+\left[\begin{array}{ccc}
A_{11} & A_{71} & A_{81} \\
A_{17} & A_{77} & A_{87} \\
A_{18} & A_{78} & A_{88}
\end{array}\right] .
$$

Here, the coefficients $A_{11}, A_{71}, A_{81}, A_{17}, A_{77}, A_{87}, A_{18}, A_{78}$, and $A_{88}$ are given as

$$
\begin{aligned}
& A_{11}=\frac{2 h+15 k-9}{2}\left(\hat{u}_{x}^{2}+\hat{u}_{y}^{2}\right) \\
& A_{71}=\frac{4 b-3}{2}\left(\hat{u}_{x}^{2}-\hat{u}_{y}^{2}\right), \quad A_{81}=2 b \hat{u}_{x} \hat{u}_{y} \\
& A_{17}=\frac{-2 h+3 k-9}{2}\left(\hat{u}_{x}^{2}-\hat{u}_{y}^{2}\right) \\
& A_{77}=\frac{-4 b-3}{2}\left(\hat{u}_{x}^{2}+\hat{u}_{y}^{2}\right), \quad A_{87}=0 \\
& A_{18}=4(h+3 k) \hat{u}_{x} \hat{u}_{y}, \quad A_{78}=0, \quad A_{88}=2 b\left(\hat{u}_{x}^{2}+\hat{u}_{y}^{2}\right) .
\end{aligned}
$$

With $R_{11}, R_{17}$, and $R_{18}$, the nonzero element $\mathbf{T}_{1}$ in the matrix $\mathbf{T}$ is determined by

$$
\begin{aligned}
\mathbf{T}_{1}= & -\frac{2-s_{e}}{2} \mathbf{B}_{1}+\frac{2-s_{e}}{4} R_{11} \mathbf{B}_{1}+\frac{2-s_{p}}{4} R_{17} \mathbf{B}_{7} \\
& +\frac{2-s_{p}}{4} R_{18} \mathbf{B}_{8}
\end{aligned}
$$

where the coefficients $\mathbf{B}_{1}, \mathbf{B}_{7}$, and $\mathbf{B}_{8}$ are given as

$$
\begin{aligned}
& \mathbf{B}_{1}=\left[\begin{array}{l}
3(k-1) \hat{u}_{x}^{3}-3\left(1+\alpha_{1}\right)(h+2 k) \hat{u}_{x} \hat{u}_{y}^{2} \\
3(k-1) \hat{u}_{y}^{3}-3\left(1+\alpha_{1}\right)(h+2 k) \hat{u}_{x}^{2} \hat{u}_{y}
\end{array}\right], \\
& \mathbf{B}_{7}=\left[\begin{array}{c}
-\hat{u}_{x}^{3}+2\left(1+\alpha_{1}\right) b \hat{u}_{x} \hat{u}_{y}^{2} \\
\hat{u}_{y}^{3}-2\left(1+\alpha_{1}\right) b \hat{u}_{x}^{2} \hat{u}_{y}
\end{array}\right], \\
& \mathbf{B}_{8}=\left[\begin{array}{l}
-\left(1+\alpha_{1}\right) b \hat{u}_{y}\left(2 \hat{u}_{x}^{2}+\hat{u}_{y}^{2}\right) \\
-\left(1+\alpha_{1}\right) b \hat{u}_{x}\left(\hat{u}_{x}^{2}+2 \hat{u}_{y}^{2}\right)
\end{array}\right] .
\end{aligned}
$$

With $R_{11}, R_{17}$, and $R_{18}$, the nonzero element $\mathbf{X}_{1}$ in the matrix $\mathbf{X}$ is determined by

$$
\begin{aligned}
\mathbf{X}_{1}= & -\frac{2-s_{e}}{2} \mathbf{C}_{1}+\frac{2-s_{e}}{4} R_{11} \mathbf{C}_{1}+\frac{2-s_{p}}{4} R_{17} \mathbf{C}_{7} \\
& +\frac{2-s_{p}}{4} R_{18} \mathbf{C}_{8},
\end{aligned}
$$

where the coefficients $\mathbf{C}_{1}, \mathbf{C}_{7}$, and $\mathbf{C}_{8}$ are given as

$$
\begin{aligned}
& \mathbf{C}_{1}=\left[\begin{array}{l}
-3 \beta_{1}(h+2 k) \hat{u}_{x} \hat{u}_{y}^{2} \\
-3 \beta_{1}(h+2 k) \hat{u}_{x}^{2} \hat{u}_{y}
\end{array}\right], \\
& \mathbf{C}_{7}=\left[\begin{array}{c}
2 \beta_{1} b \hat{u}_{x} \hat{u}_{y}^{2} \\
-2 \beta_{1} b \hat{u}_{x}^{2} \hat{u}_{y}
\end{array}\right], \\
& \mathbf{C}_{8}=\left[\begin{array}{l}
-\beta_{1} b \hat{u}_{y}\left(2 \hat{u}_{x}^{2}+\hat{u}_{y}^{2}\right) \\
-\beta_{1} b \hat{u}_{x}\left(\hat{u}_{x}^{2}+2 \hat{u}_{y}^{2}\right)
\end{array}\right] .
\end{aligned}
$$

Note that all the coefficients in Eqs. (21), (23), and (25) are locally determined by the velocity $\hat{\mathbf{u}}(\hat{\mathbf{u}} \equiv \mathbf{u} / c)$, implying that the computations of the nonzero elements $R_{11}, R_{17}, R_{18}, \mathbf{T}_{1}$, and $\mathbf{X}_{1}$ can be locally performed.

Similar to $R_{11}, R_{17}$, and $R_{18}$, the nonzero elements $R_{71}, R_{77}$, and $R_{78}$ are determined by

$$
\boldsymbol{\Psi}\left[\begin{array}{l}
\left(2-s_{e}\right) R_{71} \\
\left(2-s_{p}\right) R_{77} \\
\left(2-s_{p}\right) R_{78}
\end{array}\right]=2\left(2-s_{p}\right)\left[\begin{array}{l}
A_{71} \\
A_{77} \\
A_{78}
\end{array}\right]
$$

With $R_{71}, R_{77}$, and $R_{78}$, the nonzero elements $\mathbf{T}_{7}$ and $\mathbf{X}_{7}$ are given as

$$
\begin{aligned}
\mathbf{T}_{7}= & -\frac{2-s_{p}}{2} \mathbf{B}_{7}+\frac{2-s_{e}}{4} R_{71} \mathbf{B}_{1}+\frac{2-s_{p}}{4} R_{77} \mathbf{B}_{7} \\
& +\frac{2-s_{p}}{4} R_{78} \mathbf{B}_{8} \\
\mathbf{X}_{7}= & -\frac{2-s_{p}}{2} \mathbf{C}_{7}+\frac{2-s_{e}}{4} R_{71} \mathbf{C}_{1}+\frac{2-s_{p}}{4} R_{77} \mathbf{C}_{7} \\
& +\frac{2-s_{p}}{4} R_{78} \mathbf{C}_{8}
\end{aligned}
$$

The nonzero elements $R_{81}, R_{87}$, and $R_{88}$ are determined by

$$
\boldsymbol{\Psi}\left[\begin{array}{c}
\left(2-s_{e}\right) R_{81} \\
\left(2-s_{p}\right) R_{87} \\
\left(2-s_{p}\right) R_{88}
\end{array}\right]=2\left(2-s_{p}\right)\left[\begin{array}{l}
A_{81} \\
A_{87} \\
A_{88}
\end{array}\right]
$$

and the nonzero elements $\mathbf{T}_{8}$ and $\mathbf{X}_{8}$ are given as

$$
\begin{aligned}
\mathbf{T}_{8}= & -\frac{2-s_{p}}{2} \mathbf{B}_{8}+\frac{2-s_{e}}{4} R_{81} \mathbf{B}_{1}+\frac{2-s_{p}}{4} R_{87} \mathbf{B}_{7} \\
& +\frac{2-s_{p}}{4} R_{88} \mathbf{B}_{8},
\end{aligned}
$$




$$
\begin{aligned}
\mathbf{X}_{8}= & -\frac{2-s_{p}}{2} \mathbf{C}_{8}+\frac{2-s_{e}}{4} R_{81} \mathbf{C}_{1}+\frac{2-s_{p}}{4} R_{87} \mathbf{C}_{7} \\
& +\frac{2-s_{p}}{4} R_{88} \mathbf{C}_{8} .
\end{aligned}
$$

In practical applications, the inverse matrix of $\boldsymbol{\Psi}$ is first computed, and then all the nonzero elements in the correction matrices $\mathbf{R}, \mathbf{T}$, and $\mathbf{X}$ can be easily and locally determined by Eqs. (19), (22), (24), (26), and (27). As can be seen from Eq. (20), the matrix $\boldsymbol{\Psi}$ consists of two parts: the first part is a diagonal matrix and of order $|\hat{\mathbf{u}}|^{0}$, while the second part is a full matrix and of order $|\hat{\mathbf{u}}|^{2}$. It is very interesting to note that when the $|\hat{\mathbf{u}}|^{2}$-order terms in Eq. (20) (i.e., the second part of $\boldsymbol{\Psi}$ ) are ignored, the nonzero elements in $\mathbf{R}, \mathbf{T}$, and $\mathbf{X}$ determined by Eqs. (19), (22), (24), (26), and (27) are similar to those in the original work by Huang et al. [17], which indicates that the present improved scheme can degenerate into the previous schemes proposed under the approximations $\mathbf{R} \sim \mathcal{O}\left(\mathrm{Ma}_{\text {lattice }}^{2}\right), \mathbf{T} \sim \mathcal{O}\left(\mathrm{Ma}_{\text {lattice }}^{3}\right)$, and $\mathbf{X} \sim \mathcal{O}\left(\mathrm{Ma}_{\text {lattice }}^{3}\right)$ in Refs. [34,36,37].

\section{B. Local scheme for density gradient}

In the original work by Huang et al. [17], the density gradient $\nabla \rho$ involved in the LB equation for density DF is calculated by the conventional finite-difference scheme [i.e., the second-order isotropic central scheme (ICS)], which violates the localizability of the collision process. In this section, a local scheme will be proposed to calculate the density gradient $\nabla \rho$, which retains the advantage of the local collision process. Keeping in mind that the correction terms $-\mathbf{R}\left(\mathbf{I}-\frac{\mathbf{S}}{2}\right)\left(\mathbf{m}-\mathbf{m}^{\mathrm{eq}}+\frac{\delta_{t}}{2} \mathbf{F}_{m}\right)-\delta_{x} \mathbf{T} \cdot \nabla \rho-\delta_{x} \mathbf{X} \cdot \nabla \eta$ on the right-hand side of Eq. (2b) are aimed at eliminating the cubic terms of velocity in recovering the viscous stress tensor, we can, therefore, calculate $\nabla \rho$ and $\nabla \eta$ in these correction terms with the low lattice Mach number condition. To derive a local scheme for $\nabla \rho$ and $\nabla \eta$, the momentum conservation equation [see Eq. (10)] can be simplified as

$$
-\nabla p_{\mathrm{LBE}}+\mathbf{F}=\mathbf{0},
$$

where all the velocity-related terms are ignored. Considering $p_{\mathrm{LBE}}=p$ and $\nabla p=\left(\frac{\partial p}{\partial \rho}\right)_{T} \nabla \rho+\left(\frac{\partial p}{\partial T}\right)_{\rho} \nabla T$, Eq. (28) can be further written as

$$
\left(\frac{\partial p}{\partial \rho}\right)_{T} \nabla \rho+\left(\frac{\partial p}{\partial T}\right)_{\rho} \nabla T=\mathbf{F} .
$$

As it is well known, the temperature gradient can be locally calculated from the knowledge of the DF when the energy conservation equation is solved by the LB method [38]. Based on this principle and from the Chapman-Enskog analysis of the LB equation for total energy DF (see Appendix A in Ref. [17]), a local scheme for temperature gradient $\nabla T$ can be easily obtained:

$$
\nabla T=-\frac{6}{4+3 \gamma_{1}+2 \gamma_{2}} \frac{\sigma_{j}}{\rho_{\text {ref }} c_{\text {ref }} \delta_{x}}\left[\begin{array}{l}
n_{3}^{\text {neq }}+\frac{\sigma_{q}}{2} n_{4}^{\text {neq }} \\
n_{5}^{\text {neq }}+\frac{\sigma_{q}}{2} n_{6}^{\text {neq }}
\end{array}\right],
$$

where $\mathbf{n}^{\text {neq }}$ denotes the nonequilibrium part of $\mathbf{n}$ defined as

$$
\mathbf{n}^{\text {neq }}=\mathbf{n}-\mathbf{n}^{\mathrm{eq}}+\frac{\delta_{t}}{2} \mathbf{q}_{m} .
$$

It is noteworthy that the present definition of the nonequilibrium part is different from the previous definition $\mathbf{n}^{\text {neq }}=$ $\mathbf{n}-\mathbf{n}^{\text {eq }}$ [39] when the discrete source term exists. Once the temperature gradient $\nabla T$ is locally calculated by Eq. (30), a local scheme for density gradient $\nabla \rho$ can be easily obtained from Eq. (29) as follows:

$$
\nabla \rho=\frac{\mathbf{F}-\left(\frac{\partial p}{\partial T}\right)_{\rho} \nabla T}{\left(\frac{\partial p}{\partial \rho}\right)_{T}} .
$$

In practical applications, the EOS for fluid $p(\rho, T)$ is directly specified, and thus $\left(\frac{\partial p}{\partial T}\right)_{\rho}$ and $\left(\frac{\partial p}{\partial \rho}\right)_{T}$ can be easily obtained. Considering the relation $p_{\mathrm{LBE}}=c_{s}^{2}\left[\left(2+\alpha_{1}\right) \rho+\beta_{1} \eta\right]$, a local scheme for $\nabla \eta$ can also be derived from Eq. (28) as follows:

$$
\nabla \eta=\frac{c_{s}^{-2} \mathbf{F}-\left(2+\alpha_{1}\right) \nabla \rho}{\beta_{1}}
$$

where $\nabla \rho$ is locally calculated by Eq. (32). Here, it is worth pointing out that in the present local scheme for $\nabla \rho, \nabla T$ is first computed, which is an important quantity related to the heat flux and commonly needs to be evaluated for thermal flow problems.

\section{Scaling factor in lattice sound speed}

In the original work by Huang et al. [17], the lattice sound speed is set as

$$
c_{s}=\left.\sqrt{\left(\frac{\partial p}{\partial \rho}\right)_{T}}\right|_{\rho=\rho_{0}, T=T_{0}} .
$$

Following the idea of the LB model with self-tuning EOS for multiphase flows [34], a scaling factor $K_{\mathrm{INT}}$ is introduced into the lattice sound speed for the present DDF LB model for thermal flows, and thus $c_{s}$ is given as

$$
c_{s}=\left.K_{\mathrm{INT}} \sqrt{\left(\frac{\partial p}{\partial \rho}\right)_{T}}\right|_{\rho=\rho_{0}, T=T_{0}} .
$$

Based on our numerical tests, the scaling factor $K_{\text {INT }}$ can be adjusted to effectively enhance the numerical stability. In practical applications, the lattice spacing is determined by the computational domain and grid size, the lattice sound speed is calculated via Eq. (35), and thus the time step is given as $\delta_{t}=\sqrt{3} \delta_{x} /\left(3 c_{s}\right)$.

\section{VALIDATIONS AND DISCUSSIONS}

In this section, the thermal Couette flow, where the analytical solution exists, is first simulated to validate the present improvements in three aspects. A nonattracting rigid-sphere fluid described by the Carnahan-Starling EOS is considered. Then, the present improved LB model is applied to the simulation of the turbulent Rayleigh-Bénard convection in a supercritical fluid slightly above its critical point, which is described by the van der Waals EOS. In the simulations, both the velocity and temperature boundary conditions are treated by the method proposed in Ref. [17] for the coupled thermohydrodynamic flows. The parameter $\varpi$ is fixed at $\frac{1}{6}$, and the relaxation parameters for the density DF in $\mathbf{S}$ are determined 
under the constraints $s_{0}=s_{j}=1,\left(s_{p}^{-1}-0.5\right)\left(s_{q}^{-1}-0.5\right)=$ $\frac{1}{12},\left(s_{p}^{-1}-0.5\right) /\left(s_{e}^{-1}-0.5\right)=\varpi v / \varsigma, s_{\varepsilon}=s_{e}$, and $s_{p}=\tau_{f}^{-1}$. Here, $\tau_{f}$ is the dimensionless relaxation time for the density DF. The relaxation parameters for the total energy $\mathrm{DF}$ in $\mathbf{L}$ are determined under the constraints $\sigma_{0}=$ $1,\left(\sigma_{j}^{-1}-0.5\right)\left(\sigma_{e}^{-1}-0.5\right)=\frac{1}{4}, \sigma_{\varepsilon}=\sigma_{p}=\sigma_{e}, \sigma_{q}=\sigma_{j}$, and $\sigma_{j}=\tau_{g}^{-1}$. Here, $\tau_{g}$ is the dimensionless relaxation time for the total energy DF.

\section{A. Thermal Couette flow}

The thermal Couette flow between two parallel walls along the $x$ direction is first simulated to validate the present improvements in three aspects. The upper wall slides along the $x$ direction with a constant velocity $U_{0}$, and its temperature is fixed at $T_{0}$. The lower wall remains stationary and is kept to be adiabatic. The space between the upper and lower walls is filled with a nonattracting rigid-sphere fluid, which is described by the Carnahan-Starling EOS [40]

$$
p=Z \rho R_{g} T \quad \text { with } \quad Z=\frac{1+\vartheta+\vartheta^{2}-\vartheta^{3}}{(1-\vartheta)^{3}} .
$$

Here, $Z$ is the compressibility factor, $\vartheta=b \rho / 4$ is the packing fraction, and the maximum value of $\vartheta$ is $\sqrt{2} \pi / 6$ that corresponds to the closest-packed state. Note that the $b$ in the packing fraction $\vartheta$ should not be confused with the $b$ in the collision matrix $\mathbf{S}$. Initially, the fluid in the channel is at rest with density $\rho_{0}$ and temperature $T_{0}$. The dynamic viscosity $\mu$ and heat conductivity $\lambda$ linearly vary with the temperature as follows: $\mu / \mu_{0}=T / T_{0}$ and $\lambda / \lambda_{0}=T / T_{0}$. Under these conditions, the analytical solutions for density, velocity, and temperature can be derived as [41]

$$
\begin{gathered}
\int_{0}^{1} \frac{\rho}{\rho_{0}} d \frac{y}{W}=1 \quad \text { with } Z \rho R_{g} T=p_{\infty}, \\
\frac{u_{x}}{U_{0}}+\frac{\operatorname{Pr}_{0} \mathrm{Ma}_{e}^{2}}{2}\left(\frac{u_{x}}{U_{0}}-\frac{u_{x}^{3}}{3 U_{0}^{3}}\right)=\left(1+\frac{\operatorname{Pr}_{0} \mathrm{Ma}_{e}^{2}}{3}\right) \frac{y}{W}, \\
\frac{T}{T_{0}}=1+\frac{\operatorname{Pr}_{0} \mathrm{Ma}_{e}^{2}}{2}\left(1-\frac{u_{x}^{2}}{U_{0}^{2}}\right),
\end{gathered}
$$

where $W$ is the distance between the parallel walls, $p_{\infty}$ is the constant final pressure, $\operatorname{Pr}_{0}=c_{p, 0} \mu_{0} / \lambda_{0}$ is the Prandtl number at the temperature $T_{0}$, and $\mathrm{Ma}_{e}=U_{0} / \sqrt{c_{p, 0} T_{0}}$ is an equivalent Mach number different from the lattice Mach number $\mathrm{Ma}_{\text {lattice }}=U_{0} / c_{s}$. Here, $c_{p, 0}$ denotes the constant-pressure specific heat at the temperature $T_{0}$.

In thermodynamic theory, the general relation for a small variation in the specific internal energy can be written as [42]

$$
d \epsilon=c_{v} d T+\left[T\left(\frac{\partial p}{\partial T}\right)_{v}-p\right] d v,
$$

where $c_{v}$ is the constant-volume specific heat, and $v=1 / \rho$ is the specific volume. With the help of the Carnahan-Starling EOS, and assuming that $c_{v}$ keeps constant, a thermodynamically consistent relation between the specific internal energy $\epsilon$ and the temperature $T$ can be derived from Eq. (38):

$$
\epsilon=c_{v} T \text {. }
$$

The Mayer relation between the constant-pressure and constant-volume specific heats can be written as [42]

$$
c_{p}-c_{v}=\frac{v T \beta_{T}^{2}}{\alpha_{p}},
$$

where $\alpha_{p}=-\frac{1}{v}\left(\frac{\partial v}{\partial p}\right)_{T}$ is the isothermal compressibility, and $\beta_{T}=\frac{1}{v}\left(\frac{\partial v}{\partial T}\right)_{p}$ is the volume expansivity. With the help of the Carnahan-Starling EOS, Eq. (40) can be simplified as

$$
c_{p}-c_{v}=R_{g} \frac{Z^{2}}{Z+\rho Z^{\prime}} \quad \text { with } \quad Z^{\prime}=\frac{d Z}{d \rho} .
$$

As it can be seen, $c_{p}$ varies with $\rho$ in thermodynamic theory for the nonattracting rigid-sphere fluid described by the Carnahan-Starling EOS. When $\rho \rightarrow 0$ (i.e., the mean free path tends to infinity), Eq. (41) degenerates into $c_{p}-c_{v}=R_{g}$ for the ideal gas.

In the simulations, the channel width is set to $W=1$ and divided into 64 grids, i.e., $\delta_{x}=W / 64$. The specific gas constant is fixed at $R_{g}=1$, and the initial state of the fluid is taken as $\rho_{0}=1, T_{0}=1$, and $\vartheta\left(\rho_{0}\right)=\sqrt{2} \pi / 12$. The Prandtl number and specific heat ratio at the temperature $T_{0}$ are set to $\operatorname{Pr}_{0}=2$ and $c_{p, 0} / c_{v}=1.1$, respectively. The bulk-to-shear viscosity ratio is fixed at $\zeta / \nu=1$, and the dimensionless relaxation time for the density DF is chosen as $\tau_{f}=0.8$ for $\nu_{0}=\mu_{0} / \rho_{0}$ unless otherwise stated. The parameters in the equilibrium moment function for the total energy DF [i.e., Eq. (14)] are set to $\gamma_{1}=-2, \gamma_{2}=2, \rho_{\text {ref }}=\rho_{0}$, and $c_{\text {ref }}=c_{p, 0}$, respectively. Numerical tests are first performed to validate the present improved scheme for eliminating the cubic terms of velocity. For comparison, the original scheme in Ref. [17] is also adopted, which can be easily implemented in the present framework by ignoring the $|\hat{\mathbf{u}}|^{2}$-order terms in Eq. (20). In addition, the local scheme is employed to calculate $\nabla \rho$ and $\nabla \eta$, and the scaling factor in the lattice sound speed is set to $K_{\mathrm{INT}}=1$. Figure 2 shows the distributions of the density $\rho / \rho_{0}$, velocity $u_{x} / U_{0}$, and temperature $T / T_{0}$ with the coordinate $y / W$ for the equivalent Mach number $\mathrm{Ma}_{e}=0.1,0.2,0.3$, and 0.4, respectively. As it can be seen, the numerical results by the improved scheme agree very well with the analytical solutions, while the numerical results by the original scheme obviously deviate from the analytical solutions when $\mathrm{Ma}_{e}=0.3$ and 0.4 . Thus, the present improved scheme for eliminating the cubic terms of velocity is more accurate than the original one in Ref. [17]. To quantitatively demonstrate the higher accuracy of the improved scheme, the relative errors are calculated, which are defined as

$$
E_{\phi}=\sqrt{\frac{\sum\left[f(\phi)_{\text {numerical }}-f(\phi)_{\text {analytical }}\right]^{2}}{\sum f(\phi)_{\text {analytical }}^{2}}},
$$

where $f(\phi)$ denotes $\rho / \rho_{0}, u_{x} / U_{0}$, and $T / T_{0}$ when $\phi=\rho, u$, and $T$, respectively, and the subscripts "numerical" and "analytical" represent the numerical result and analytical solution of $f(\phi)$, respectively. The variations of $E_{\rho}, E_{u}$, and $E_{T}$ with $\mathrm{Ma}_{e}$ in the range $0.01 \leqslant \mathrm{Ma}_{e} \leqslant 0.64$ are shown in Fig. 3. As it can be seen, the relative errors by the improved scheme are much smaller than those by the original scheme, and the gaps tend to become larger as $\mathrm{Ma}_{e}$ increases. Quantitatively, for $\mathrm{Ma}_{e}=0.01, E_{\rho}$ by the improved scheme is the same as $E_{\rho}$ by 


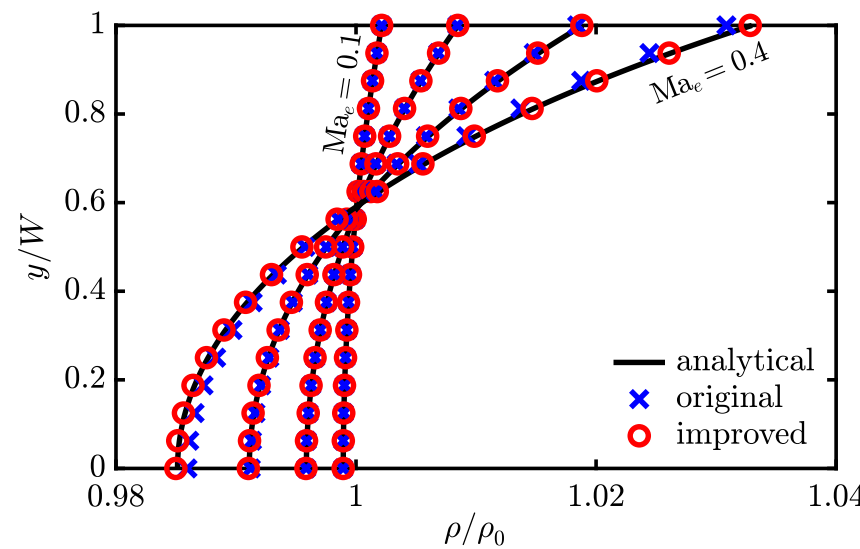

(a)

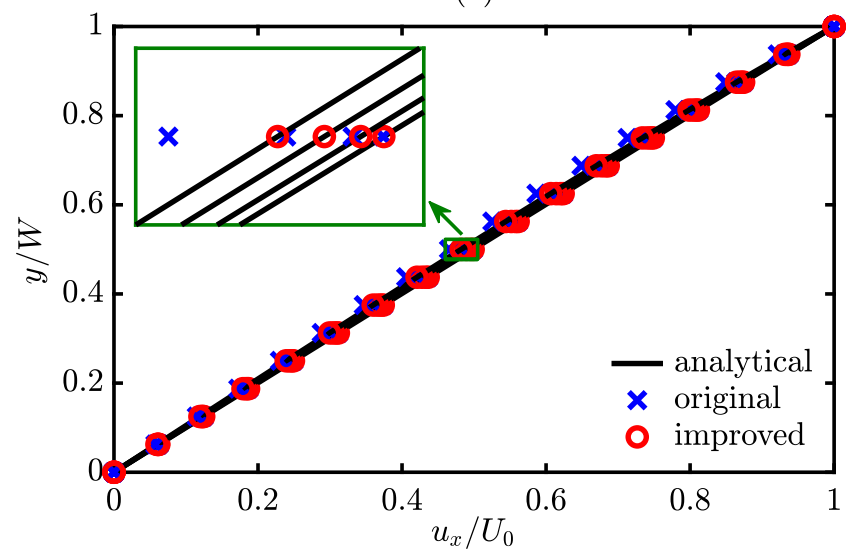

(b)

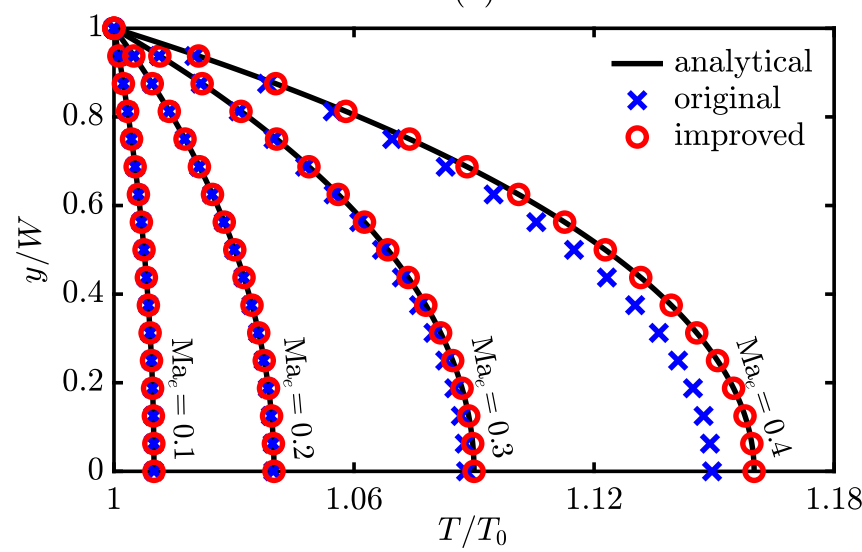

(c)

FIG. 2. Distributions of the density $\rho / \rho_{0}$, velocity $u_{x} / U_{0}$, and temperature $T / T_{0}$ with the coordinate $y / W$ for $\mathrm{Ma}_{e}=0.1,0.2,0.3$, and 0.4 , respectively. The present improved scheme and the original scheme in Ref. [17] are adopted to eliminate the cubic terms of velocity, the local scheme is employed to calculate $\nabla \rho$ and $\nabla \eta$, and the scaling factor in the lattice sound speed is set to $K_{\mathrm{INT}}=1$.

the original scheme, while $E_{u}$ and $E_{T}$ by the improved scheme are $3.087 \%$ and $17.756 \%$ of $E_{u}$ and $E_{T}$ by the original scheme, respectively. As $\mathrm{Ma}_{e}$ increases to $0.64, E_{\rho}, E_{u}$, and $E_{T}$ by the improved scheme are only $3.780 \%, 0.0657 \%$, and $0.0465 \%$ of $E_{\rho}, E_{u}$, and $E_{T}$ by the original scheme, respectively.

To specifically validate the present local scheme for density gradient, the improved scheme is employed to eliminate the

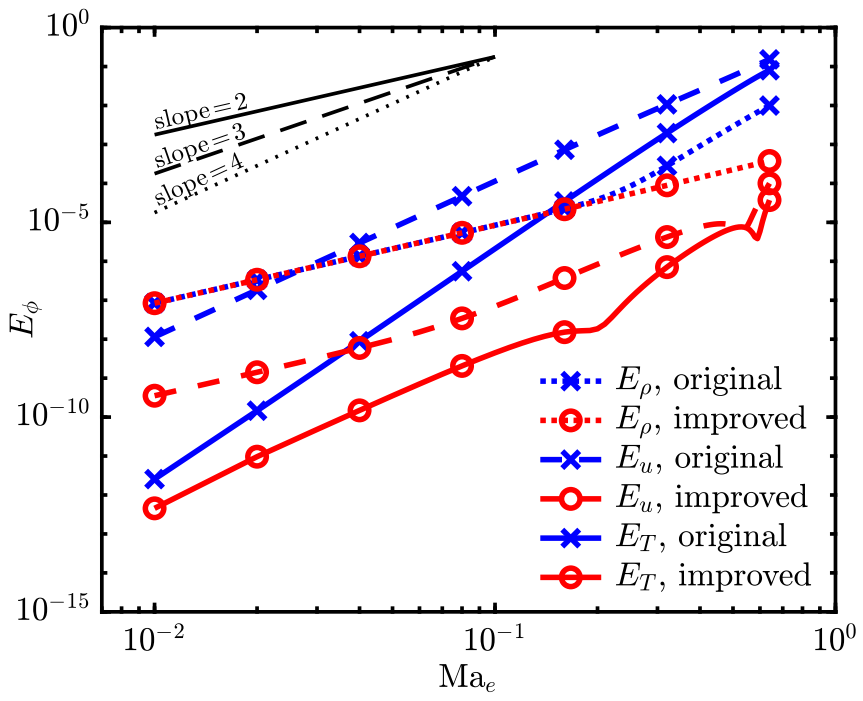

FIG. 3. Variations of the relative errors $E_{\rho}, E_{u}$, and $E_{T}$ with the equivalent Mach number $\mathrm{Ma}_{e}$ in the range $0.01 \leqslant \mathrm{Ma}_{e} \leqslant 0.64$. The present improved scheme and the original scheme in Ref. [17] are adopted to eliminate the cubic terms of velocity, the local scheme is employed to calculate $\nabla \rho$ and $\nabla \eta$, and the scaling factor in the lattice sound speed is set to $K_{\mathrm{INT}}=1$.

cubic terms of velocity, the present local scheme is employed to calculate $\nabla \rho$ and $\nabla \eta$, and the scaling factor in the lattice sound speed is set to $K_{\mathrm{INT}}=1$ in the simulations. For the sake of comparison, the density gradient is also calculated by the second-order ICS based on the numerical profile of the density. Figure 4 shows the distributions of the density derivative $\partial_{y} \rho$ with the coordinate $y / W$ for the equivalent Mach number $\mathrm{Ma}_{e}=0.1,0.2,0.3$, and 0.4 , respectively. It can be seen from Fig. 4 that the numerical result by the local scheme agrees very well with the analytical solution, and there is no discernible difference in the results by the local scheme and the second-order ICS. These good agreements demonstrate the applicability and accuracy of the present local

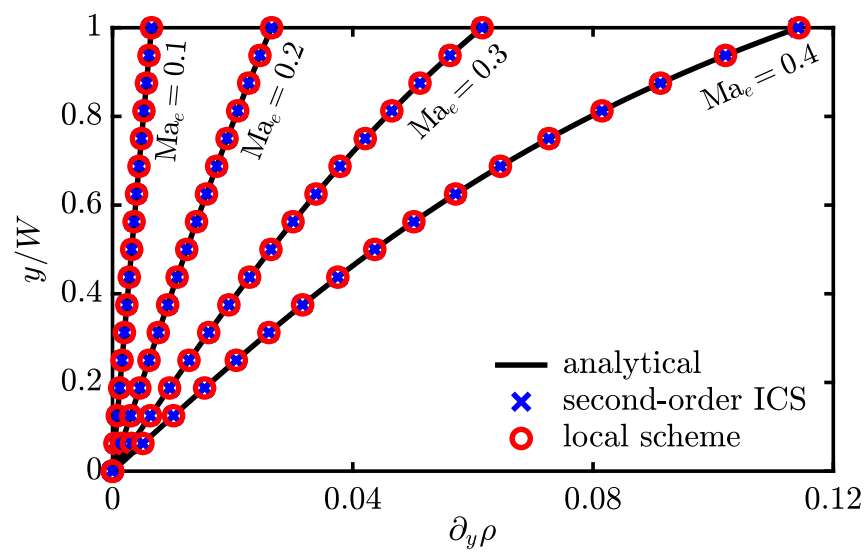

FIG. 4. Distributions of the density derivative $\partial_{y} \rho$ with the coordinate $y / W$ for $\mathrm{Ma}_{e}=0.1,0.2,0.3$, and 0.4 , respectively. The improved scheme is employed to eliminate the cubic terms of velocity, the local scheme is employed to calculate $\nabla \rho$ and $\nabla \eta$, and the scaling factor in the lattice sound speed is set to $K_{\mathrm{INT}}=1$. 


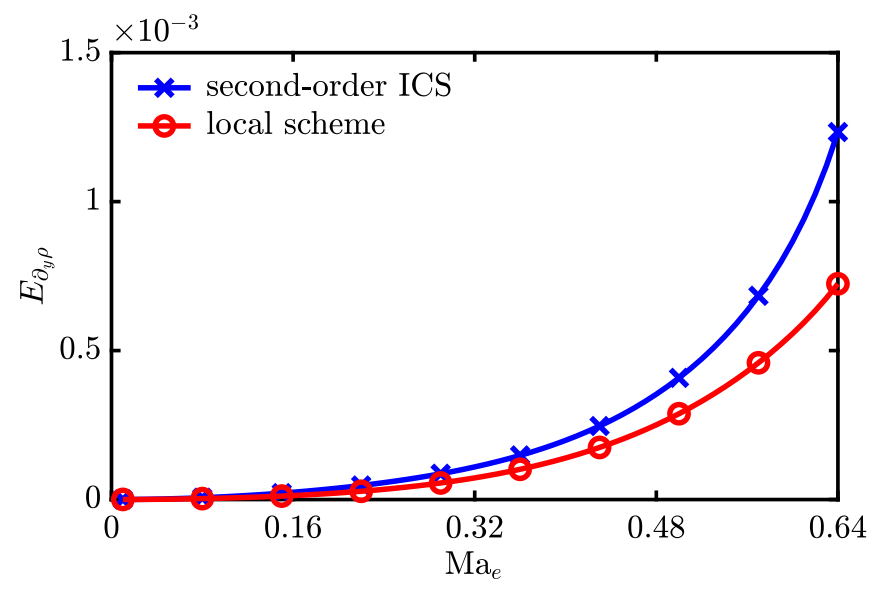

FIG. 5. Variations of the relative error $E_{\partial y} \rho$ with the equivalent Mach number $\mathrm{Ma}_{e}$ in the range $0.01 \leqslant \mathrm{Ma}_{e} \leqslant 0.64$. The improved scheme is employed to eliminate the cubic terms of velocity, the local scheme is employed to calculate $\nabla \rho$ and $\nabla \eta$, and the scaling factor in the lattice sound speed is set to $K_{\mathrm{INT}}=1$.

scheme for density gradient. To further compare the local scheme and the second-order ICS, the relative error of $\partial_{y} \rho$ is calculated, which is also defined by Eq. (42) $[f(\phi)$ denotes $\partial_{y} \rho$ when $\left.\phi=\partial_{y} \rho\right]$. The variations of $E_{\partial_{y} \rho}$ with $\mathrm{Ma}_{e}$ in the range $0.01 \leqslant \mathrm{Ma}_{e} \leqslant 0.64$ are shown in Fig. 5. It is interesting to observe from Fig. 5 that $E_{\partial_{y} \rho}$ by the local scheme is always smaller than that by the second-order ICS, and the mean ratio of $E_{\partial y \rho}$ by the local scheme to that by the second-order ICS is about 0.630 . Thus, as compared with the second-order ICS, the present local scheme not only retains the advantage of the local collision process, but also shows improvement in numerical accuracy.

In the following, the effect of the present scaling factor $K_{\mathrm{INT}}$ introduced into the lattice sound speed is numerically investigated. For this purpose, the improved scheme is employed to eliminate the cubic terms of velocity, and the local scheme is employed to calculate $\nabla \rho$ and $\nabla \eta$. The scaling factor in the lattice sound speed is set to $K_{\mathrm{INT}}=1,2$, and 3 , respectively, and the dimensionless relaxation time for the density DF is chosen as $\tau_{f}=0.5+0.3 / K_{\mathrm{INT}}$ for $\nu_{0}=$ $\mu_{0} / \rho_{0}$ to ensure that the kinematic viscosity $\left[v=c_{s} \delta_{x}\left(\tau_{f}-\right.\right.$ $0.5) / \sqrt{3}$ ] remains unchanged in the simulations. Figure 6 shows the distributions of density $\rho / \rho_{0}$, velocity $u_{x} / U_{0}$, and temperature $T / T_{0}$ with the coordinate $y / W$ for $\mathrm{Ma}_{e}=0.4$, $0.7,1.0$, and 1.3, respectively. As seen in Fig. 6, the results obtained with different $K_{\mathrm{INT}}$ are indistinguishable as long as the simulations are stable, implying that the adjustment of $K_{\text {INT }}$ does not deteriorate the numerical accuracy. The simulation with $K_{\mathrm{INT}}=1$ loses numerical stability for $\mathrm{Ma}_{e}=0.7,1.0$, and 1.3 , while the simulation with $K_{\mathrm{INT}}=2$ loses numerical stability only for $\mathrm{Ma}_{e}=1.3$. However, the simulation with $K_{\mathrm{INT}}=3$ keeps stable for all the involved $\mathrm{Ma}_{e}$. Figure 7 gives the maximum $\mathrm{Ma}_{e}$ (denoted by $\mathrm{Ma}_{e, \text { max }}$ ) that can be achieved in the simulation with different $K_{\mathrm{INT}}$ in the range $0.1 \leqslant K_{\mathrm{INT}} \leqslant 10$. It can be seen from Fig. 7 that the simulation blows up when $K_{\mathrm{INT}} \leqslant 0.6$, and $\mathrm{Ma}_{e, \text { max }}$ increases as $K_{\mathrm{INT}}$ increases in the range $0.7 \leqslant K_{\mathrm{INT}} \leqslant 5.3$. However, in the range $K_{\mathrm{INT}} \geqslant 5.4, \mathrm{Ma}_{e, \max }$ decreases as $K_{\mathrm{INT}}$ increases,

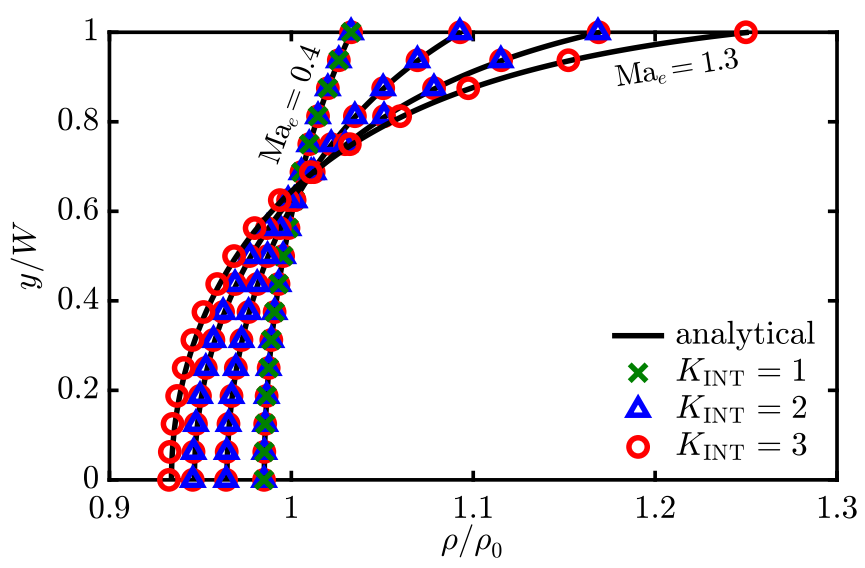

(a)

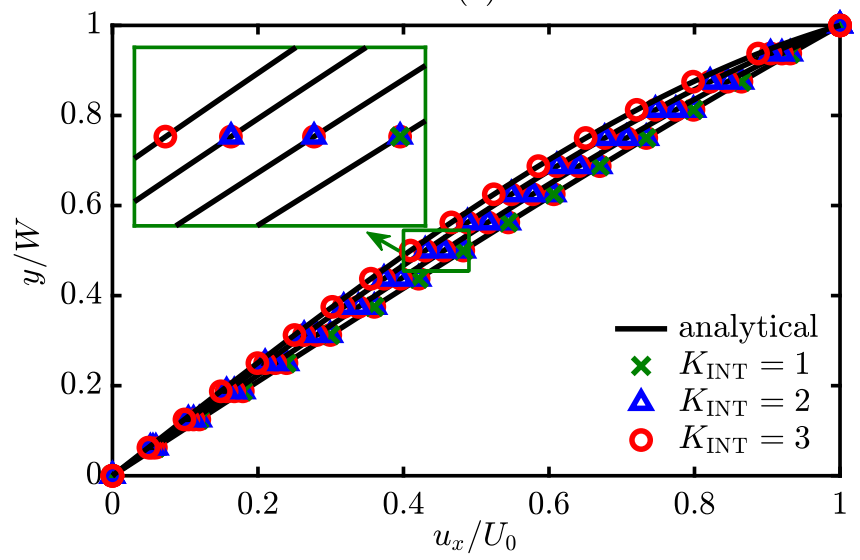

(b)

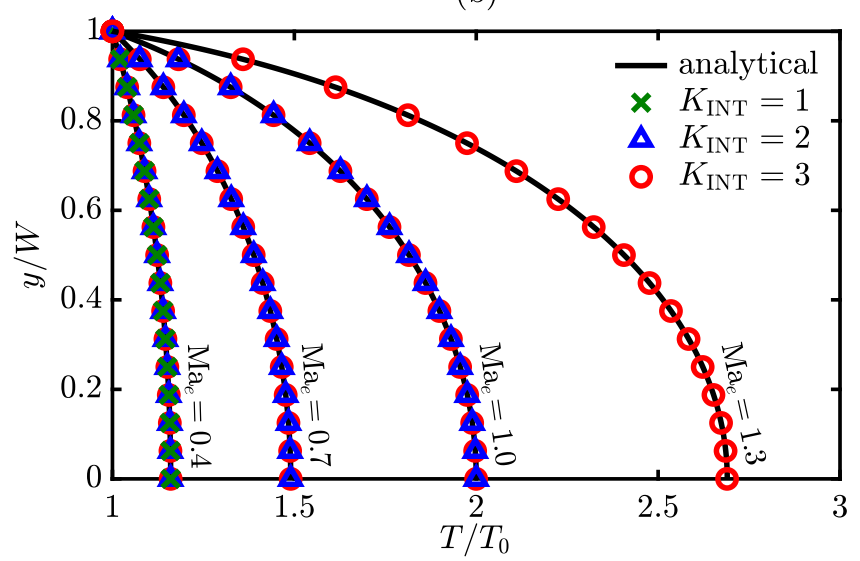

(c)

FIG. 6. Distributions of the density $\rho / \rho_{0}$, velocity $u_{x} / U_{0}$, and temperature $T / T_{0}$ with the coordinate $y / W$ for $\mathrm{Ma}_{e}=0.4,0.7,1.0$, and 1.3 , respectively. The improved scheme is employed to eliminate the cubic terms of velocity, the local scheme is employed to calculate $\nabla \rho$ and $\nabla \eta$, and the scaling factor in the lattice sound speed is set to $K_{\mathrm{INT}}=1,2$, and 3 , respectively.

which is probably caused by the fact that $\tau_{f}$ tends to 0.5 when $K_{\mathrm{INT}}$ is relatively large. Nevertheless, increasing the scaling factor $K_{\mathrm{INT}}$ in an appropriate range can effectively enhance the numerical stability and thus help to achieve larger $\mathrm{Ma}_{e}$ in the simulation. Here, it is worth pointing out that both the lattice Mach number $\mathrm{Ma}_{\text {lattice }}$ and the time step $\delta_{t}$ decrease 


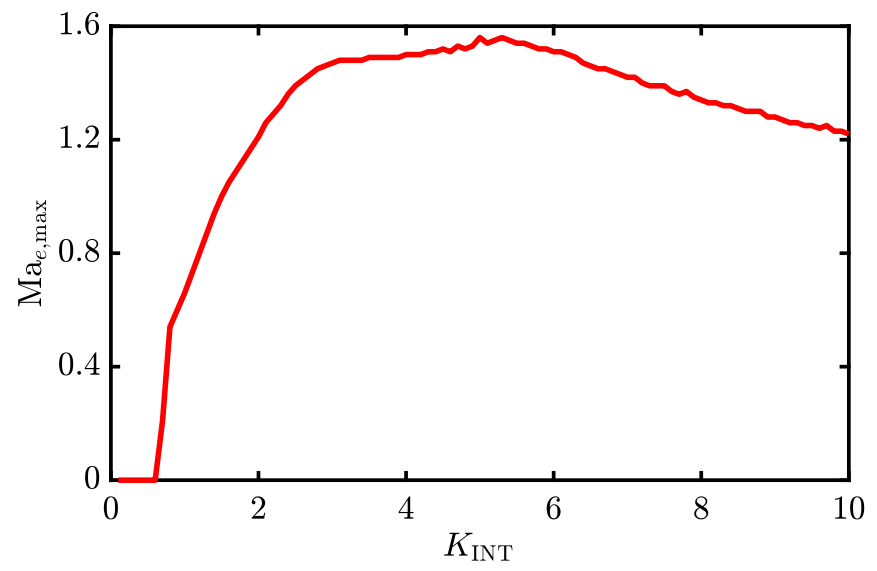

FIG. 7. Variation of the maximum achieved equivalent Mach number $\mathrm{Ma}_{e, \max }$ with the scaling factor in lattice sound speed $K_{\mathrm{INT}}$ in the range $0.1 \leqslant K_{\mathrm{INT}} \leqslant 10$. The improved scheme is employed to eliminate the cubic terms of velocity, the local scheme is employed to calculate $\nabla \rho$ and $\nabla \eta$, and the scaling factor in the lattice sound speed is adjusted from $K_{\mathrm{INT}}=0.1$ to 10 .

as $K_{\text {INT }}$ increases, which indicates that the computational cost becomes higher when $K_{\mathrm{INT}}$ increases.

\section{B. Rayleigh-Bénard convection}

In this section, the present improved LB model with selftuning EOS is applied to the simulation of the turbulent Rayleigh-Bénard convection in a supercritical fluid slightly above its critical point, as illustrated by Fig. 8. Once the supercritical fluid slightly above its critical point is heated from the bottom wall, a hot thermal boundary layer (TBL) will be formed along the bottom wall and the fluid in this layer will significantly expand due to the critical divergence of the volume expansivity $\left[\beta_{T}=\frac{1}{v}\left(\frac{\partial v}{\partial T}\right)_{p}\right]$. Such expansion will adiabatically compress the fluid in the bulk region and thus induce a fast and homogeneous increase of the temperature in the bulk region, which is called the piston effect and is first observed by Nitsche and Straub under the microgravity condition in 1987 [43]. Since the temperature of the top wall is kept at the initial temperature, a cold TBL will also be formed along the top wall. Thus, at the early stage of the heating process, the supercritical fluid in the channel can be divided into three regions: the hot and cold TBLs and the bulk region, as shown in Fig. 8. As time goes on, the TBLs

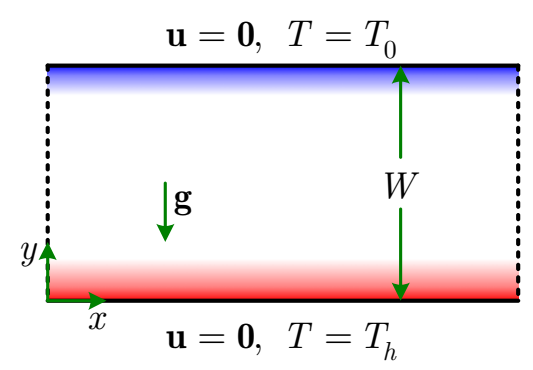

FIG. 8. Illustration of the Rayleigh-Bénard convection in a supercritical fluid slightly above its critical point. gradually develop, and the Rayleigh-Bénard instability will be triggered.

In this work, the classical van der Waals EOS is adopted to describe the supercritical fluid

$$
p=\frac{\rho R_{g} T}{1-b \rho}-a \rho^{2},
$$

where the coefficients $a$ and $b$ are related to the critical point as

$$
a=\frac{9 R_{g} T_{\mathrm{cr}}}{8 \rho_{\mathrm{cr}}}, \quad b=\frac{1}{3 \rho_{\mathrm{cr}}} .
$$

Here, $\rho_{\mathrm{cr}}$ and $T_{\mathrm{cr}}$ denote the critical density and temperature, respectively, and the critical pressure is given as $p_{\mathrm{cr}}=$ $3 \rho_{\mathrm{cr}} R_{g} T_{\mathrm{cr}} / 8$. Based on the general thermodynamic relation given by Eq. (38), a thermodynamically consistent relation between the specific internal energy $\epsilon$ and the temperature $T$ can be derived as follows:

$$
\epsilon=c_{v} T-a \rho,
$$

where the constant-volume specific heat $c_{v}$ is assumed to be constant. Based on the Mayer relation given by Eq. (40), the constant-pressure specific heat $c_{p}$ can be determined as

$$
c_{p}=c_{v}+R_{g} \frac{R_{g} T}{R_{g} T-2 a \rho(1-b \rho)^{2}} .
$$

It can be seen from Eq. (46) that $c_{p}$ varies with both $\rho$ and $T$ for the supercritical fluid described by the van der Waals EOS although $c_{v}$ is constant. The heat conductivity $\lambda$ varies with the temperature $T$ as follows:

$$
\lambda=\lambda_{\mathrm{IG}}\left(1+0.75 \varepsilon^{-0.5}\right),
$$

where $\lambda_{\mathrm{IG}}$ refers to the heat conductivity for the ideal gas, and $\varepsilon=T / T_{\mathrm{cr}}-1$ defines the dimensionless proximity to the critical point. The dynamic viscosity $\mu$ is assumed to be constant

$$
\mu=\mu_{\mathrm{IG}}
$$

where $\mu_{\mathrm{IG}}$ refers to the dynamic viscosity for the ideal gas.

Due to the critical divergence of the isothermal compressibility $\left[\alpha_{p}=-\frac{1}{v}\left(\frac{\partial v}{\partial p}\right)_{T}\right]$, the supercritical fluid slightly above its critical point in the channel could be obviously stratified in density under the effect of its own weight. Thus, special care should be paid to the initialization of the simulation. For this purpose, the supercritical fluid in the channel is first set to be at rest with uniform density $\rho_{0}\left(\rho_{0}=\rho_{c}\right)$ and temperature $T_{0}$ $\left[T_{0}=\left(1+\varepsilon_{0}\right) T_{\text {cr }}\right.$ with $\left.\varepsilon_{0} \ll 1\right]$, and both the temperatures of the bottom and top walls are fixed at $T_{0}$. Then, an auxiliary simulation of the stratification process is run to a steady state, which is the correct initialization of the heating process. From this moment on (denoted as $t=0$ ), the temperature of the bottom wall is linearly raised to $T_{h}\left(T_{h}>T_{0}\right)$ during the time $0 \leqslant t \leqslant t_{\mathrm{PE}}$ and then kept at $T_{h}$, while the temperature of the top wall is always maintained at the initial temperature $T_{0}$. Here, $t_{\mathrm{PE}}$ is the piston-effect timescale defined as [44]

$$
t_{\mathrm{PE}}=\frac{\rho_{\mathrm{cr}} W^{2}}{\varepsilon_{0}^{-1}\left(0.75^{-1}+\varepsilon_{0}^{-0.5}\right) \mu_{\mathrm{IG}}},
$$

where $W$ is the channel width. Generally, the pistoneffect timescale is much larger than the thermal diffusion 


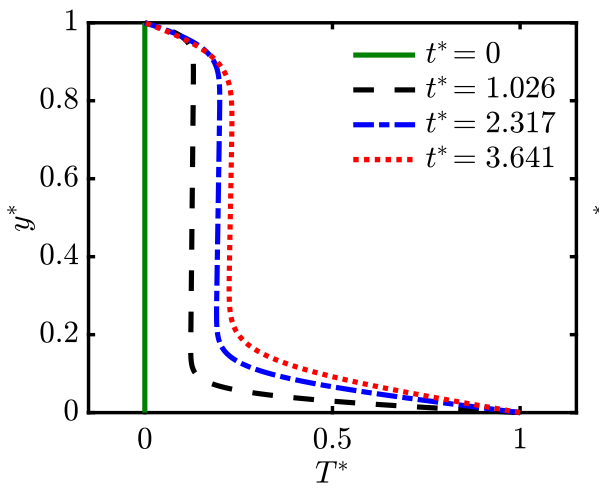

(a)

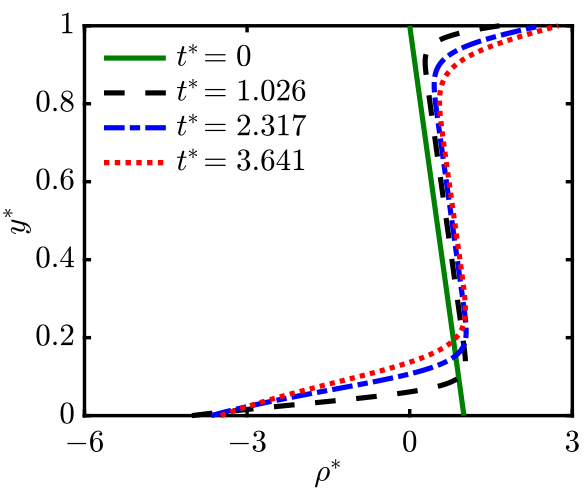

(b)

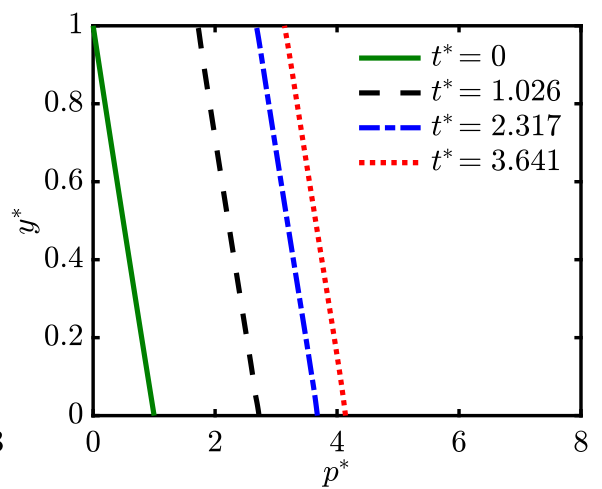

(c)

FIG. 9. Distributions of the temperature $T^{*}$, density $\rho^{*}$, and pressure $p^{*}$ with the coordinate $y^{*}$ at time $t^{*}=0,1.026,2.317$, and 3.641, respectively. The numerical results remain to be one dimensional since the Rayleigh-Bénard instability has not been triggered at the early stage.

timescale, which indicates that energy is transferred much faster by piston effect than by heat conduction. In the simulation, the thermophysical properties are taken as $T_{\mathrm{cr}}=$ $304.13 \mathrm{~K}, \rho_{\mathrm{cr}}=467.8 \mathrm{~kg} / \mathrm{m}^{3}, R_{g}=188 \mathrm{~J} /(\mathrm{kg} \mathrm{K})$, and $c_{v}=$ $658 \mathrm{~J} /(\mathrm{kg} \mathrm{K})$, and the gravity acceleration is $|\mathbf{g}|=9.81 \mathrm{~m} / \mathrm{s}^{2}$. The initial temperature is set to $T_{0}=314.13 \mathrm{~K}$, implying that $\varepsilon_{0}=0.0328807$, and the heating temperature is set to $T_{h}=$ $324.13 \mathrm{~K}$. Two dimensionless parameters, the Prandtl number $\mathrm{Pr}_{\mathrm{IG}}$ and the Froude number Fr, are introduced and defined as

$$
\operatorname{Pr}_{\mathrm{IG}}=\frac{c_{p, \mathrm{IG}} \mu_{\mathrm{IG}}}{\lambda_{\mathrm{IG}}}, \quad \mathrm{Fr}=\frac{U_{\mathrm{PE}}^{2}}{|\mathbf{g}| W}
$$

Here, $c_{p, \mathrm{IG}}=c_{v}+R_{g}$ is the constant-pressure specific heat for the ideal gas [see Eq. (46) when $T \rightarrow+\infty$ or $\rho \rightarrow 0$ ], and $U_{\mathrm{PE}}=W / t_{\mathrm{PE}}$ is piston-effect velocity scale. These two dimensionless parameters are taken as $\operatorname{Pr}_{\mathrm{IG}}=2.91024$ and $\mathrm{Fr}=0.01$, respectively. The lattice sound speed $c_{s}$ is calculated with the initial density $\rho_{0}$ and temperature $T_{0}$, and the scaling factor is set to $K_{\mathrm{INT}}=4$. The dimensionless relaxation time for the density DF is chosen as $\tau_{f}=0.55$ for $v=\mu_{\mathrm{IG}} / \rho_{\mathrm{cr}}$, and the viscosity ratio is fixed at $\zeta / v=4$. For the parameters in $\mathbf{n}^{\mathrm{eq}}$, the coefficients are set to $\gamma_{1}=-2.0$ and $\gamma_{2}=1.2$, respectively, and the reference density is chosen as $\rho_{\text {ref }}=\rho_{\text {cr }}$, while the reference specific heat capacity is determined by ensuring $\tau_{g}=0.9$ for $\lambda=\lambda_{\mathrm{IG}}\left(1+0.75 \varepsilon_{0}^{-0.5}\right)$. The computational domain is chosen as $2 W \times W$ and divided into $2048 \times 1024$ grids, and the periodic condition is imposed in the $x$ direction, as illustrated by Fig. 8 .

As mentioned above, the supercritical fluid in the channel is stratified in density due to its own weight at time $t=0$. Based on the auxiliary simulation of the stratification process, the fluid densities on the bottom and top walls are $\rho_{\text {bottom }}=$ $480.625942 \mathrm{~kg} / \mathrm{m}^{3}$ and $\rho_{\text {top }}=454.974931 \mathrm{~kg} / \mathrm{m}^{3}$, respectively, and the fluid pressures on the bottom and top walls are $p_{\text {bottom }}=11.404695 \mathrm{MPa}$ and $p_{\text {top }}=11.295551 \mathrm{MPa}$, respectively. For the sake of discussion, the temperature, density, and pressure are normalized as $T^{*}=(T-$ $\left.T_{0}\right) /\left(T_{h}-T_{0}\right), \quad \rho^{*}=\left(\rho-\rho_{\text {top }}\right) /\left(\rho_{\text {bottom }}-\rho_{\text {top }}\right)$, and $p^{*}=$ $\left(p-p_{\text {top }}\right) /\left(p_{\text {bottom }}-p_{\text {top }}\right)$, respectively, and the time and coordinate are normalized as $t^{*}=t / t_{\mathrm{PE}}$ and $\mathbf{x}^{*}=\mathbf{x} / W$, respectively. Figure 9 shows the distributions of the temperature $T^{*}$, density $\rho^{*}$, and pressure $p^{*}$ with the coordinate $y^{*}$ at time $t^{*}=0,1.026,2.317$, and 3.641, respectively. Note that the numerical results remain to be one dimensional since the Rayleigh-Bénard instability has not been triggered. It can be seen from Fig. 9 that at time $t^{*}=0$, the temperature $T^{*}$ uniformly remains at the value of 0 , while the density $\rho^{*}$ and pressure $p^{*}$ gradually decrease from 1 on the bottom wall $\left(y^{*}=0\right)$ to 0 on the top wall $\left(y^{*}=1\right)$. At time $t^{*}=1.026$, 2.317, and 3.641, three distinct regions, i.e., the hot TBL, the bulk region, and the cold TBL, can be clearly observed from Fig. 9(a). In the hot and cold TBLs, the temperature $T^{*}$ rapidly decreases as $y^{*}$ increases, and the thicknesses of the TBLs grow as time goes on. In the bulk region, the temperature $T^{*}$ is spatially uniform but gradually increases as time goes on, and this anomalous heating in the bulk region can be theoretically explained by the piston effect [45]. From Fig. 9(b), it can be seen that the density $\rho^{*}$ in the bulk region gradually decreases as $y^{*}$ increases due to the weight of the supercritical fluid, while the density $\rho^{*}$ in the hot and cold TBLs rapidly increases as $y^{*}$ increases, which can be explained by the critical divergence of the volume expansivity $\left[\beta_{T}=\frac{1}{v}\left(\frac{\partial v}{\partial T}\right)_{p}\right]$. Here, it is worth pointing out that the decrease of $\rho^{*}$ with $y^{*}$ in the bulk region acts to stabilize the fluid, while the increase of $\rho^{*}$ with $y^{*}$ in the TBLs could trigger the Rayleigh-Bénard instability. As clearly shown in Fig. 9(c), the distributions of the pressure $p^{*}$ with $y^{*}$ at $t^{*}=0,1.026$, 2.317, and 3.641 are similar to each other, and the mean value of $p^{*}$ gradually grows as time goes on. This is because the variation of $p^{*}$ with $y^{*}$ is caused by the fluid weight not only in the bulk region but also in the TBLs, and the mean value of $T^{*}$ continuously grows with time, which can be observed from Fig. 9(a).

The thickness of the TBL grows as time goes on, and the Rayleigh-Bénard instability will be triggered within the TBL. Figure 10 shows the temperature field $T^{*}$, together with the velocity vector every 64 points in both $x$ and $y$ directions, at time $t^{*}=3.972,4.304,4.469,4.800,5.297$, and 6.290, respectively. Simultaneously, the dimensionless heat flux (i.e., the Nusselt number $\mathrm{Nu}$ ) along the bottom wall is also plotted, 


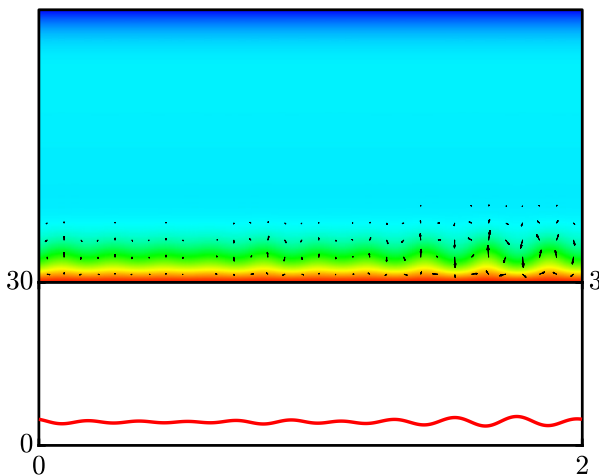

(a)

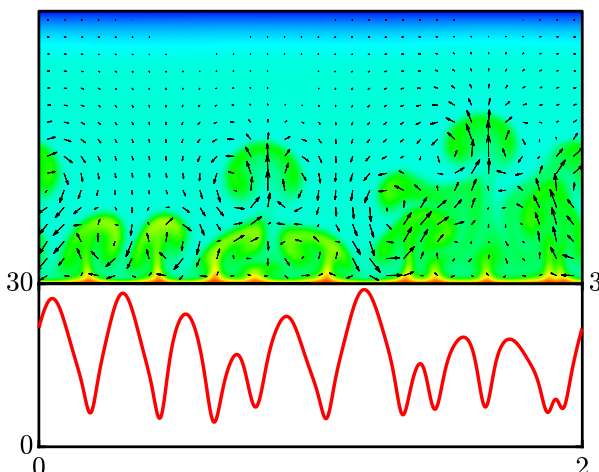

(d)

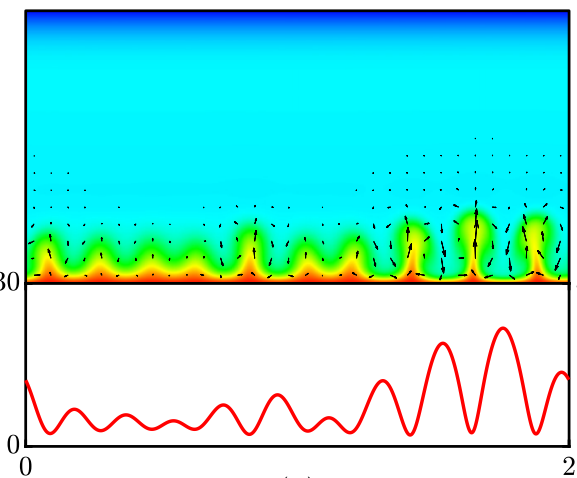

(b)

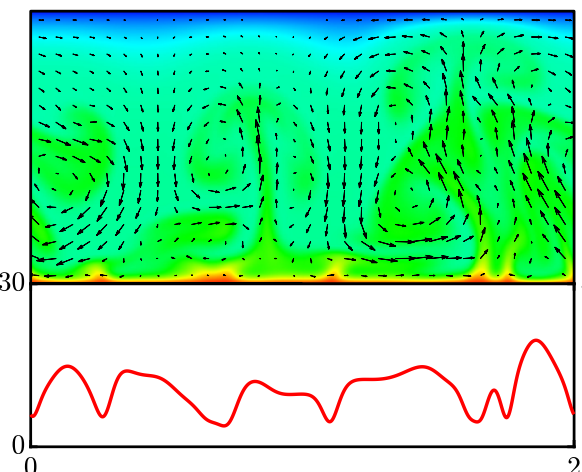

(e)

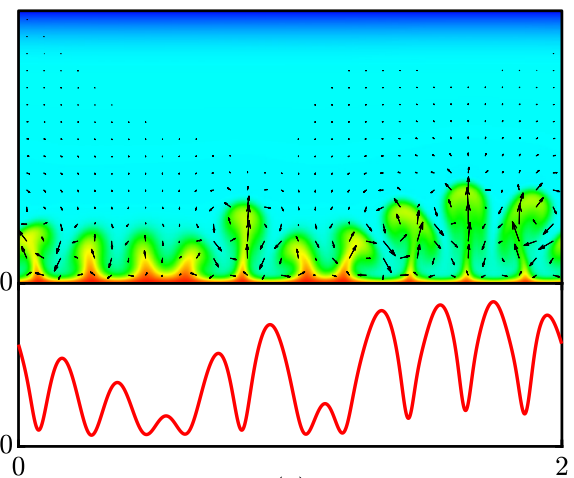

(c)

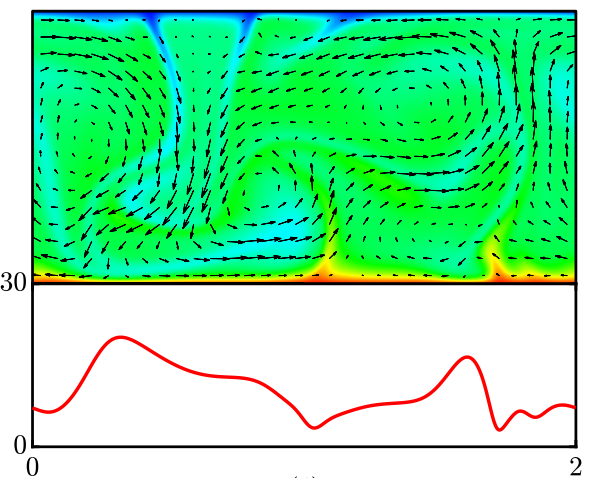

(f)

FIG. 10. Temperature field $T^{*}$, together with the velocity vector every 64 points in both $x$ and $y$ directions, and distribution of the Nusselt number $\mathrm{Nu}$ along the bottom wall at time (a) $t^{*}=3.972$, (b) $t^{*}=4.304$, (c) $t^{*}=4.469$, (d) $t^{*}=4.800$, (e) $t^{*}=5.297$, and (f) $t^{*}=6.290$, respectively.

which is defined as

$$
\mathrm{Nu}=\frac{-W \lambda_{h} \partial_{y} T}{\lambda_{0}\left(T_{h}-T_{0}\right)} .
$$

Here, $\lambda_{0}$ and $\lambda_{h}$ are the heat conductivities at the temperatures $T_{0}$ and $T_{h}$, respectively, and the temperature derivative $\partial_{y} T$ is locally computed by Eq. (30). Figure 11 shows the density field $\rho^{*}$, together with the velocity vector, at the same moments as Fig. 10. It can be seen from Figs. 10 and 11 that at time $t^{*}=3.972$, the hot TBL is slightly disturbed and the convection develops within it, while the cold TBL remains stable. This is expected because the thickness of the hot TBL

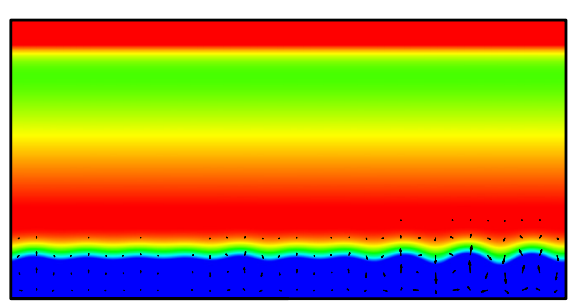

(a)

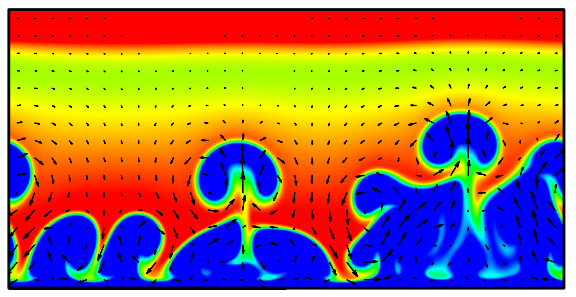

(d)

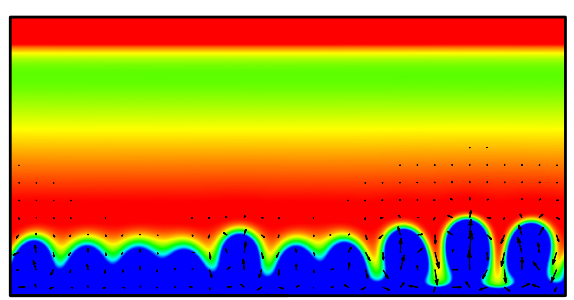

(b)

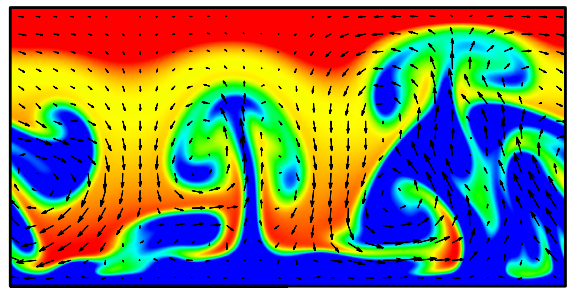

(e)

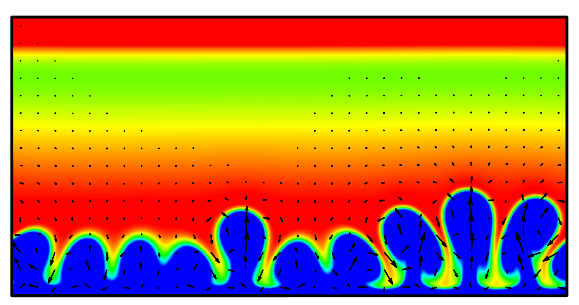

(c)

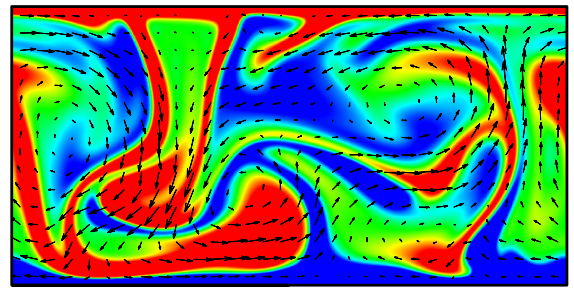

(f)

FIG. 11. Density field $\rho^{*}$, together with the velocity vector every 64 points in both $x$ and $y$ directions, at time (a) $t^{*}=3.972$, (b) $t^{*}=4.304$, (c) $t^{*}=4.469$, (d) $t^{*}=4.800$, (e) $t^{*}=5.297$, and (f) $t^{*}=6.290$, respectively. 
is greater than the cold one, and the local Rayleigh number associated with the hot TBL reaches the critical value that triggers the Rayleigh-Bénard instability at a relatively early time. At time $t^{*}=4.304$, the hot TBL is greatly disturbed and many thermal plumes are generated within it. At the site of a thermal plume, the Nusselt number $\mathrm{Nu}$ decreases as the local heat transfer is weakened, while at the site between thermal plumes, the Nusselt number $\mathrm{Nu}$ obviously increases as the local heat transfer is greatly enhanced. Along with the development of thermal plumes, the heavier fluid in the bulk region penetrates into the hot TBL, which can help to enhance the local heat transfer. At time $t^{*}=4.469$ and 4.800 , the thermal plumes detach from the hot TBL and rise under the effect of buoyancy. The detachment of a thermal plume can further enhance the local heat transfer, and the rise of a thermal plume can drive the large-scale circulation. At time $t^{*}=5.297$, the rising plume reaches the cold TBL and acts as a disturbance to the cold TBL. Consequently, the heavier fluid within the cold TBL starts falling, as seen in Fig. 11(e). In addition, the Nusselt number $\mathrm{Nu}$ along the bottom wall slightly decreases compared with that at time $t^{*}=4.800$ because the thickness of the hot TBL grows again after the detachment of thermal plumes. At time $t^{*}=6.290$, the large-scale circulation is formed, and the final turbulent state of the Rayleigh-Bénard convection in a supercritical fluid is reached. Some thermal plumes are continuously generated within both the hot and cold TBLs, and the number of plumes is much less than those at time $t^{*}=4.304,4.469$, and 4.800 because the lateral movement of the fluid near the walls is quite obvious in the final turbulent state.

To better understand the evolution of the Rayleigh-Bénard convection, the time evolutions of the average Nusselt number along the bottom wall $\mathrm{Nu}_{\text {ave }}$ and the average kinetic energy $E_{k, \text { ave }}^{*}$ are shown in Fig. 12, which are defined as

$$
\begin{aligned}
\mathrm{Nu}_{\mathrm{ave}} & =\frac{1}{2 W} \int_{0}^{2 W} \mathrm{Nu} d x, \\
E_{k, \text { ave }}^{*} & =\frac{1}{2 W^{2}} \int_{0}^{2 W} \int_{0}^{W} \frac{\rho|\mathbf{u}|^{2} / 2}{\rho_{\mathrm{cr}} U_{\mathrm{PE}}^{2} / 2} d x d y,
\end{aligned}
$$

where $\mathrm{Nu}$ is defined by Eq. (51), and the kinetic energy $\rho|\mathbf{u}|^{2} / 2$ is normalized by $\rho_{\mathrm{cr}} U_{\mathrm{PE}}^{2} / 2$. In this figure, the moments in Fig. 10 (the same as Fig. 11) are represented by the solid points, and the time evolution is sequentially divided into the heating, diffusion, convection, transition, and turbulence stages that are depicted by different background colors. The first stage is the heating stage with $0 \leqslant t^{*} \leqslant 1$, which means that the temperature of the bottom wall is linearly raised from $T_{0}$ to $T_{h}$. At the heating stage, the average Nusselt number $\mathrm{Nu}_{\text {ave }}$ gradually increases, while the average kinetic energy $E_{k \text {,ave }}^{*}$ remains at 0 . The second stage is the diffusion stage with $1 \leqslant t^{*} \leqslant 3.990$, which means that diffusion is the dominating heat transfer mechanism. Therefore, $\mathrm{Nu}_{\mathrm{ave}}$ gradually decreases as the thickness of the hot TBL grows, and $E_{k \text {,ave }}^{*}$ still remains at 0 . The third stage is the convection stage with $3.990 \leqslant t^{*} \leqslant 4.762$, which means that the Rayleigh-Bénard instability is triggered and the convection starts. Since the dominating heat transfer mechanism changes from diffusion to convection, $\mathrm{Nu}_{\text {ave }}$ increases rapidly, while $E_{k \text {,ave }}^{*}$ increases relatively slowly as the convection occurs locally in space at

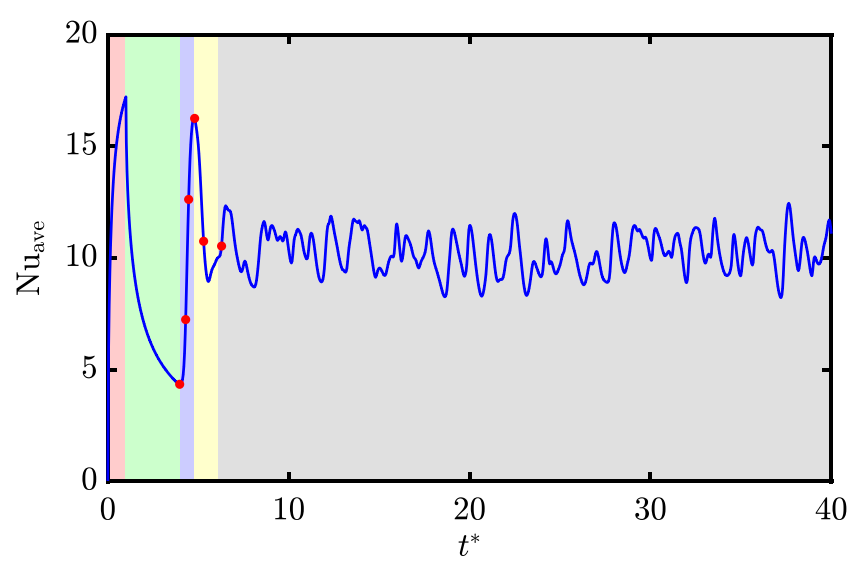

(a)

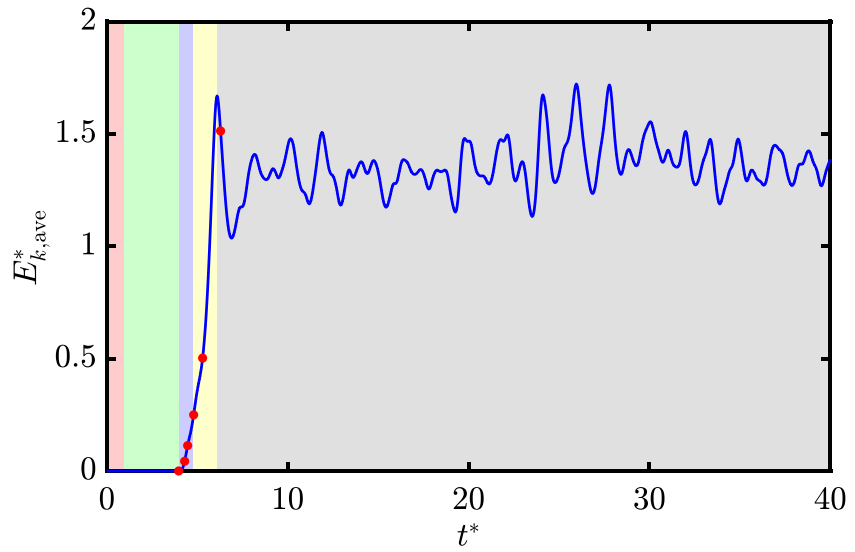

(b)

FIG. 12. Time evolutions of the average Nusselt number along the bottom wall $\mathrm{Nu}_{\text {ave }}$ and the average kinetic energy $E_{k \text {,ave }}^{*}$ The moments in Fig. 10 (the same as Fig. 11) are represented by the solid points, and the time evolution is sequentially divided into the heating, diffusion, convection, transition, and turbulence stages that are depicted by different background colors.

this stage. The fourth one is the transition stage with $4.762 \leqslant$ $t^{*} \leqslant 6.096$, which means that the Rayleigh-Bénard convection undergoes a transition to turbulence. At this stage, $\mathrm{Nu}_{\text {ave }}$ slightly decreases compared with that at the end of the convection stage, while $E_{k \text {,ave }}^{*}$ continuously and rapidly increases due to the formation of the large-scale circulation. The last stage is the turbulence stage with $t^{*} \geqslant 6.096$, which means that the Rayleigh-Bénard convection finally develops into turbulence. At this stage, both $\mathrm{Nu}_{\text {ave }}$ and $E_{k \text {,ave }}^{*}$ randomly fluctuate with time, and their time-averaged values are 10.182 and 1.347, respectively. Here, the time-averaged value of $E_{k \text {,ave }}^{*}$ is at the order of 1, implying that the magnitude of $U_{\mathrm{PE}}$ is quite close to the space-averaged value of $|\mathbf{u}|$. This confirms that the pistoneffect timescale is much larger than the thermal diffusion timescale as convection is much stronger than diffusion, and also indicates that the piston-effect timescale is much smaller than the acoustic timescale considering that the Mach number is quite low for the turbulent Rayleigh-Bénard convection in a supercritical fluid slightly above its critical point. 


\section{CONCLUSION}

This work focuses on improving the recent LB model with self-tuning EOS for the simulation of thermal flows beyond the Boussinesq and ideal-gas approximations. An improved scheme is proposed to eliminate the additional cubic terms of velocity in recovering the viscous stress tensor without any further approximations. Meanwhile, with the help of a local scheme for temperature gradient, a local scheme is proposed to calculate the density gradient instead of the second-order ICS. In addition, following the idea of the LB model for multiphase flows, a scaling factor is introduced into the lattice sound speed for the present LB model for thermal flows. The present improvements in three aspects are first validated by simulating the thermal Couette flow of a nonattracting rigid-sphere fluid described by the Carnahan-Starling EOS. Numerical results demonstrate that the improved scheme for eliminating the cubic terms of velocity can significantly improve the numerical accuracy, and the scaling factor in the lattice sound speed can be adjusted to effectively enhance the numerical stability. It is also interesting to find that the local scheme for density gradient can not only retain the advantage of the local collision process but also show improvement in numerical accuracy. Then, the present improved LB model is applied to the simulation of the turbulent Rayleigh-Bénard convection in a supercritical fluid slightly above its critical point, which is described by the van der Waals EOS. The piston effect due to the critical divergence of the volume expansivity is successfully captured, which induces a fast and homogeneous increase of the temperature in the bulk region at the early stage of the heating process. The time evolution from the initiation of heating to the final turbulent state is analyzed in detail and is sequentially divided into the heating, diffusion, convection, transition, and turbulence stages.

\section{ACKNOWLEDGMENTS}

This work was supported by the National Natural Science Foundation of China through Grants No. 52006244 and No. 51822606

\section{APPENDIX: DERIVATION OF THE IMPROVED SCHEME}

The Chapman-Enskog analysis of the LB equation for density DF is performed to reveal the additional cubic terms of velocity in recovering the viscous stress tensor and to derive the improved scheme for eliminating these cubic terms of velocity. For this purpose, performing the Taylor series expansion of $f_{i}\left(\mathbf{x}+\mathbf{e}_{i} \delta_{t}, t+\delta_{t}\right)$ centered at $(\mathbf{x}, t)$ in Eq. (2a), and then transforming the result into moment space and combining it with Eq. (2b), we can obtain

$$
\begin{aligned}
\left(\mathbf{I} \partial_{t}\right. & +\mathbf{D}) \mathbf{m}+\frac{\delta_{t}}{2}\left(\mathbf{I} \partial_{t}+\mathbf{D}\right)^{2} \mathbf{m}-\mathbf{F}_{m}+O\left(\delta_{t}^{2}\right) \\
= & -\left[\frac{\mathbf{S}}{\delta_{t}}+\frac{\mathbf{R}}{\delta_{t}}\left(\mathbf{I}-\frac{\mathbf{S}}{2}\right)\right]\left(\mathbf{m}-\mathbf{m}^{\mathrm{eq}}+\frac{\delta_{t}}{2} \mathbf{F}_{m}\right) \\
& -c \mathbf{T} \cdot \nabla \rho-c \mathbf{X} \cdot \nabla \eta,
\end{aligned}
$$

where $\mathbf{D}=\mathbf{M}\left[\operatorname{diag}\left(\mathbf{e}_{i} \cdot \nabla\right)\right] \mathbf{M}^{-1}$. With the following Chapman-Enskog expansions [34]

$$
\begin{aligned}
& \partial_{t}=\sum_{n=1}^{+\infty} \varepsilon^{n} \partial_{t n}, \quad \nabla=\varepsilon^{1} \nabla_{1}, \quad f_{i}=\sum_{n=0}^{+\infty} \varepsilon^{n} f_{i}^{(n)}, \quad \mathbf{F}=\varepsilon^{1} \mathbf{F}^{(1)}, \\
& \mathbf{D}=\varepsilon^{1} \mathbf{D}_{1}, \quad \mathbf{m}=\sum_{n=0}^{+\infty} \varepsilon^{n} \mathbf{m}^{(n)}, \quad \mathbf{F}_{m}=\varepsilon^{1} \mathbf{F}_{m}^{(1)},
\end{aligned}
$$

Eq. (A1) can be rewritten in the consecutive orders of $\varepsilon$ as follows:

$$
\begin{aligned}
& \varepsilon^{0}:-\frac{\mathbf{S}}{\delta_{t}}\left(\mathbf{m}^{(0)}-\mathbf{m}^{\mathrm{eq}}\right)-\frac{\mathbf{R}}{\delta_{t}}\left(\mathbf{I}-\frac{\mathbf{S}}{2}\right)\left(\mathbf{m}^{(0)}-\mathbf{m}^{\mathrm{eq}}\right)=\mathbf{0}, \\
& \varepsilon^{1}:\left(\mathbf{I} \partial_{t 1}+\mathbf{D}_{1}\right) \mathbf{m}^{(0)}-\mathbf{F}_{m}^{(1)}=-\frac{\mathbf{S}}{\delta_{t}} \mathbf{G}^{(1)}+\frac{2}{\delta_{t}}\left(\tilde{\mathbf{G}}^{(1)}-\hat{\mathbf{G}}^{(1)}\right), \\
& \varepsilon^{2}: \partial_{t 2} \mathbf{m}^{(0)}+\left(\mathbf{I} \partial_{t 1}+\mathbf{D}_{1}\right) \tilde{\mathbf{G}}^{(1)}=-\frac{\mathbf{S}}{\delta_{t}} \mathbf{m}^{(2)}-\frac{\mathbf{R}}{\delta_{t}}\left(\mathbf{I}-\frac{\mathbf{S}}{2}\right) \mathbf{m}^{(2)} .
\end{aligned}
$$

Here, $\varepsilon$ is the small Chapman-Enskog expansion parameter, and $\mathbf{G}^{(1)} \equiv \mathbf{m}^{(1)}+\frac{\delta_{t}}{2} \mathbf{F}_{m}^{(1)}, \hat{\mathbf{G}}^{(1)} \equiv\left(\mathbf{I}-\frac{\mathbf{S}}{2}\right) \mathbf{G}^{(1)}$, and $\tilde{\mathbf{G}}^{(1)} \equiv$ $\left(\mathbf{I}-\frac{\mathbf{R}}{2}\right) \hat{\mathbf{G}}^{(1)}-\frac{\delta_{x}}{2} \mathbf{T} \cdot \nabla_{1} \rho-\frac{\delta_{x}}{2} \mathbf{X} \cdot \nabla_{1} \eta$ are introduced to simplify the notations.

To concentrate on eliminating the cubic terms of velocity, the lengthy and repetitious calculations of the ChapmanEnskog analysis will not be covered here, and the reader is referred to Ref. [34]. We start with Eqs. (19), (21), and (23) in Ref. [34], where the additional cubic terms are revealed. Substituting the relation $p_{\mathrm{LBE}}=c_{s}^{2}\left[\left(2+\alpha_{1}\right) \rho+\beta_{1} \eta\right]$ into Eqs. (19), (21), and (23) in Ref. [34], we can reformulate these equations as follows:

$$
\begin{aligned}
& -\frac{1}{\delta_{t}} \frac{2 s_{e}}{2-s_{e}} \hat{G}_{1}^{(1)}+\frac{2}{\delta_{t}}\left(\tilde{G}_{1}^{(1)}-\hat{G}_{1}^{(1)}\right)=2 \varpi c \rho\left(\nabla_{1} \cdot \hat{\mathbf{u}}\right)+c\left[\begin{array}{l}
A_{11} \rho\left(\nabla_{1} \cdot \hat{\mathbf{u}}\right)+A_{17} \rho\left(\partial_{x 1} \hat{u}_{x}-\partial_{y 1} \hat{u}_{y}\right) \\
+A_{18} \rho\left(\partial_{x 1} \hat{u}_{y}+\partial_{y 1} \hat{u}_{x}\right)+\mathbf{B}_{1} \cdot \nabla_{1} \rho+\mathbf{C}_{1} \cdot \nabla_{1} \eta+O_{1}\left(|\hat{\mathbf{u}}|^{5}\right)
\end{array}\right], \\
& -\frac{1}{\delta_{t}} \frac{2 s_{p}}{2-s_{p}} \hat{G}_{7}^{(1)}+\frac{2}{\delta_{t}}\left(\tilde{G}_{7}^{(1)}-\hat{G}_{7}^{(1)}\right)=\frac{2}{3} c \rho\left(\partial_{x 1} \hat{u}_{x}-\partial_{y 1} \hat{u}_{y}\right)+c\left[\begin{array}{l}
A_{71} \rho\left(\nabla_{1} \cdot \hat{\mathbf{u}}\right)+A_{77} \rho\left(\partial_{x 1} \hat{u}_{x}-\partial_{y 1} \hat{u}_{y}\right) \\
+A_{78} \rho\left(\partial_{x 1} \hat{u}_{y}+\partial_{y 1} \hat{u}_{x}\right)+\mathbf{B}_{7} \cdot \nabla_{1} \rho+\mathbf{C}_{7} \cdot \nabla_{1} \eta+O_{7}\left(|\hat{\mathbf{u}}|^{5}\right)
\end{array}\right], \\
& -\frac{1}{\delta_{t}} \frac{2 s_{p}}{2-s_{p}} \hat{G}_{8}^{(1)}+\frac{2}{\delta_{t}}\left(\tilde{G}_{8}^{(1)}-\hat{G}_{8}^{(1)}\right)=\frac{1}{3} c \rho\left(\partial_{x 1} \hat{u}_{y}+\partial_{y 1} \hat{u}_{x}\right)+c\left[\begin{array}{l}
A_{81} \rho\left(\nabla_{1} \cdot \hat{\mathbf{u}}\right)+A_{87} \rho\left(\partial_{x 1} \hat{u}_{x}-\partial_{y 1} \hat{u}_{y}\right) \\
+A_{88} \rho\left(\partial_{x 1} \hat{u}_{y}+\partial_{y 1} \hat{u}_{x}\right)+\mathbf{B}_{8} \cdot \nabla_{1} \rho+\mathbf{C}_{8} \cdot \nabla_{1} \eta+O_{8}\left(|\hat{\mathbf{u}}|^{5}\right)
\end{array}\right],
\end{aligned}
$$


where the coefficients on the right-hand sides are given by Eqs. (21), (23), and (25), and the fifth-order terms $O_{1}\left(|\hat{\mathbf{u}}|^{5}\right)$, $O_{7}\left(|\hat{\mathbf{u}}|^{5}\right)$, and $O_{8}\left(|\hat{\mathbf{u}}|^{5}\right)$ can be ignored with the low lattice Mach number condition. The viscous stress tensor recovered through the Chapman-Enskog analysis can be generally written as $[17,34]$

$\Pi=\varepsilon^{1} \boldsymbol{\Pi}^{(1)}=-\varepsilon^{1} c^{2}\left[\begin{array}{cc}\frac{1}{2} \tilde{G}_{7}^{(1)} & \tilde{G}_{8}^{(1)} \\ \tilde{G}_{8}^{(1)} & -\frac{1}{2} \tilde{G}_{7}^{(1)}\end{array}\right]-\varepsilon^{1} c^{2}\left[\begin{array}{cc}\frac{1}{6} \tilde{G}_{1}^{(1)} & 0 \\ 0 & \frac{1}{6} \tilde{G}_{1}^{(1)}\end{array}\right]$

(A5)
Therefore, to eliminate the additional cubic terms in recovering the viscous stress tensor, there should have

$$
\begin{aligned}
-\frac{1}{\delta_{t}} \frac{2 s_{e}}{2-s_{e}} \tilde{G}_{1}^{(1)} & =2 \varpi c \rho\left(\nabla_{1} \cdot \hat{\mathbf{u}}\right), \\
-\frac{1}{\delta_{t}} \frac{2 s_{p}}{2-s_{p}} \tilde{G}_{7}^{(1)} & =\frac{2}{3} c \rho\left(\partial_{x 1} \hat{u}_{x}-\partial_{y 1} \hat{u}_{y}\right), \\
-\frac{1}{\delta_{t}} \frac{2 s_{p}}{2-s_{p}} \tilde{G}_{8}^{(1)} & =\frac{1}{3} c \rho\left(\partial_{x 1} \hat{u}_{y}+\partial_{y 1} \hat{u}_{x}\right) .
\end{aligned}
$$

Substituting Eqs. (A6a), (A6b), and (A6c) into the left-hand sides of Eqs. (A4a), (A4b), and (A4c), respectively, we have

$$
\begin{aligned}
& \hat{G}_{1}^{(1)}=-\frac{2-s_{e}}{2 s_{e}} 2 \varpi \delta_{x} \rho\left(\nabla_{1} \cdot \hat{\mathbf{u}}\right)-\frac{2-s_{e}}{4} \delta_{x}\left[\begin{array}{l}
A_{11} \rho\left(\nabla_{1} \cdot \hat{\mathbf{u}}\right)+A_{17} \rho\left(\partial_{x 1} \hat{u}_{x}-\partial_{y 1} \hat{u}_{y}\right) \\
+A_{18} \rho\left(\partial_{x 1} \hat{u}_{y}+\partial_{y 1} \hat{u}_{x}\right)+\mathbf{B}_{1} \cdot \nabla_{1} \rho+\mathbf{C}_{1} \cdot \nabla_{1} \eta
\end{array}\right], \\
& \hat{G}_{7}^{(1)}=-\frac{2-s_{p}}{2 s_{p}} \frac{2}{3} \delta_{x} \rho\left(\partial_{x 1} \hat{u}_{x}-\partial_{y 1} \hat{u}_{y}\right)-\frac{2-s_{p}}{4} \delta_{x}\left[\begin{array}{l}
A_{71} \rho\left(\nabla_{1} \cdot \hat{\mathbf{u}}\right)+A_{77} \rho\left(\partial_{x 1} \hat{u}_{x}-\partial_{y 1} \hat{u}_{y}\right) \\
+A_{78} \rho\left(\partial_{x 1} \hat{u}_{y}+\partial_{y 1} \hat{u}_{x}\right)+\mathbf{B}_{7} \cdot \nabla_{1} \rho+\mathbf{C}_{7} \cdot \nabla_{1} \eta
\end{array}\right], \\
& \hat{G}_{8}^{(1)}=-\frac{2-s_{p}}{2 s_{p}} \frac{1}{3} \delta_{x} \rho\left(\partial_{x 1} \hat{u}_{y}+\partial_{y 1} \hat{u}_{x}\right)-\frac{2-s_{p}}{4} \delta_{x}\left[\begin{array}{l}
A_{81} \rho\left(\nabla_{1} \cdot \hat{\mathbf{u}}\right)+A_{87} \rho\left(\partial_{x 1} \hat{u}_{x}-\partial_{y 1} \hat{u}_{y}\right) \\
+A_{88} \rho\left(\partial_{x 1} \hat{u}_{y}+\partial_{y 1} \hat{u}_{x}\right)+\mathbf{B}_{8} \cdot \nabla_{1} \rho+\mathbf{C}_{8} \cdot \nabla_{1} \eta
\end{array}\right] .
\end{aligned}
$$

Substituting Eq. (A6a) into the right-hand side of Eq. (A4a), we can get

$$
\tilde{G}_{1}^{(1)}-\hat{G}_{1}^{(1)}=\frac{2-s_{e}}{4} \delta_{x}\left[\begin{array}{l}
A_{11} \rho\left(\nabla_{1} \cdot \hat{\mathbf{u}}\right)+A_{17} \rho\left(\partial_{x 1} \hat{u}_{x}-\partial_{y 1} \hat{u}_{y}\right) \\
+A_{18} \rho\left(\partial_{x 1} \hat{u}_{y}+\partial_{y 1} \hat{u}_{x}\right)+\mathbf{B}_{1} \cdot \nabla_{1} \rho+\mathbf{C}_{1} \cdot \nabla_{1} \eta
\end{array}\right] .
$$

With the help of $\tilde{\mathbf{G}}^{(1)}-\hat{\mathbf{G}}^{(1)}=-\frac{\mathbf{R}}{2} \hat{\mathbf{G}}^{(1)}-\frac{\delta_{x}}{2} \mathbf{T} \cdot \nabla_{1} \rho-\frac{\delta_{x}}{2} \mathbf{X} \cdot \nabla_{1} \eta$, Eq. (A8) can be further written as

$$
R_{11} \hat{G}_{1}^{(1)}+R_{17} \hat{G}_{7}^{(1)}+R_{18} \hat{G}_{8}^{(1)}+\delta_{x} \mathbf{T}_{1} \cdot \nabla_{1} \rho+\delta_{x} \mathbf{X}_{1} \cdot \nabla_{1} \eta=-\frac{2-s_{e}}{2} \delta_{x}\left[\begin{array}{c}
A_{11} \rho\left(\nabla_{1} \cdot \hat{\mathbf{u}}\right)+A_{17} \rho\left(\partial_{x 1} \hat{u}_{x}-\partial_{y 1} \hat{u}_{y}\right) \\
+A_{18} \rho\left(\partial_{x 1} \hat{u}_{y}+\partial_{y 1} \hat{u}_{x}\right)+\mathbf{B}_{1} \cdot \nabla_{1} \rho+\mathbf{C}_{1} \cdot \nabla_{1} \eta
\end{array}\right] .
$$

Substituting Eq. (A7) into the left-hand side of Eq. (A9), and then equating the coefficients of the same terms on the left- and right-hand sides of the result, the nonzero elements $R_{11}, R_{17}, R_{18}, \mathbf{T}_{1}$, and $\mathbf{X}_{1}$ can be uniquely determined by

$$
\begin{gathered}
\boldsymbol{\Psi}\left[\begin{array}{l}
\left(2-s_{e}\right) R_{11} \\
\left(2-s_{p}\right) R_{17} \\
\left(2-s_{p}\right) R_{18}
\end{array}\right]=2\left(2-s_{e}\right)\left[\begin{array}{l}
A_{11} \\
A_{17} \\
A_{18}
\end{array}\right], \\
\mathbf{T}_{1}=-\frac{2-s_{e}}{2} \mathbf{B}_{1}+\frac{2-s_{e}}{4} R_{11} \mathbf{B}_{1}+\frac{2-s_{p}}{4} R_{17} \mathbf{B}_{7}+\frac{2-s_{p}}{4} R_{18} \mathbf{B}_{8}, \\
\mathbf{X}_{1}=-\frac{2-s_{e}}{2} \mathbf{C}_{1}+\frac{2-s_{e}}{4} R_{11} \mathbf{C}_{1}+\frac{2-s_{p}}{4} R_{17} \mathbf{C}_{7}+\frac{2-s_{p}}{4} R_{18} \mathbf{C}_{8},
\end{gathered}
$$

where $\boldsymbol{\Psi}$ is the $3 \times 3$ matrix given by Eq. (20). Similarly, substituting Eq. (A6b) into the right-hand side of Eq. (A4b), and considering $\tilde{\mathbf{G}}^{(1)}-\hat{\mathbf{G}}^{(1)}=-\frac{\mathbf{R}}{2} \hat{\mathbf{G}}^{(1)}-\frac{\delta_{x}}{2} \mathbf{T} \cdot \nabla_{1} \rho-\frac{\delta_{x}}{2} \mathbf{X} \cdot \nabla_{1} \eta$, we have

$$
R_{71} \hat{G}_{1}^{(1)}+R_{77} \hat{G}_{7}^{(1)}+R_{78} \hat{G}_{8}^{(1)}+\delta_{x} \mathbf{T}_{7} \cdot \nabla_{1} \rho+\delta_{x} \mathbf{X}_{7} \cdot \nabla_{1} \eta=-\frac{2-s_{p}}{2} \delta_{x}\left[\begin{array}{c}
A_{71} \rho\left(\nabla_{1} \cdot \hat{\mathbf{u}}\right)+A_{77} \rho\left(\partial_{x 1} \hat{u}_{x}-\partial_{y 1} \hat{u}_{y}\right) \\
+A_{78} \rho\left(\partial_{x 1} \hat{u}_{y}+\partial_{y 1} \hat{u}_{x}\right)+\mathbf{B}_{7} \cdot \nabla_{1} \rho+\mathbf{C}_{7} \cdot \nabla_{1} \eta
\end{array}\right] .
$$

Substituting Eq. (A7) into the left-hand side of Eq. (A11), and then equating the coefficients of the same terms, the nonzero elements $R_{71}, R_{77}, R_{78}, \mathbf{T}_{7}$, and $\mathbf{X}_{7}$ can be determined by

$$
\boldsymbol{\Psi}\left[\begin{array}{l}
\left(2-s_{e}\right) R_{71} \\
\left(2-s_{p}\right) R_{77} \\
\left(2-s_{p}\right) R_{78}
\end{array}\right]=2\left(2-s_{p}\right)\left[\begin{array}{l}
A_{71} \\
A_{77} \\
A_{78}
\end{array}\right],
$$

$$
\begin{aligned}
\mathbf{T}_{7}= & -\frac{2-s_{p}}{2} \mathbf{B}_{7}+\frac{2-s_{e}}{4} R_{71} \mathbf{B}_{1}+\frac{2-s_{p}}{4} R_{77} \mathbf{B}_{7} \\
& +\frac{2-s_{p}}{4} R_{78} \mathbf{B}_{8} \\
\mathbf{X}_{7}= & -\frac{2-s_{p}}{2} \mathbf{C}_{7}+\frac{2-s_{e}}{4} R_{71} \mathbf{C}_{1}+\frac{2-s_{p}}{4} R_{77} \mathbf{C}_{7} \\
& +\frac{2-s_{p}}{4} R_{78} \mathbf{C}_{8}
\end{aligned}
$$


Substituting Eq. (A6c) into the right-hand side of Eq. (A4c), and considering $\tilde{\mathbf{G}}^{(1)}-\hat{\mathbf{G}}^{(1)}=-\frac{\mathbf{R}}{2} \hat{\mathbf{G}}^{(1)}-\frac{\delta_{x}}{2} \mathbf{T} \cdot \nabla_{1} \rho-\frac{\delta_{x}}{2} \mathbf{X} \cdot \nabla_{1} \eta$, we have

$$
R_{81} \hat{G}_{1}^{(1)}+R_{87} \hat{G}_{7}^{(1)}+R_{88} \hat{G}_{8}^{(1)}+\delta_{x} \mathbf{T}_{8} \cdot \nabla_{1} \rho+\delta_{x} \mathbf{X}_{8} \cdot \nabla_{1} \eta=-\frac{2-s_{p}}{2} \delta_{x}\left[\begin{array}{c}
A_{81} \rho\left(\nabla_{1} \cdot \hat{\mathbf{u}}\right)+A_{87} \rho\left(\partial_{x 1} \hat{u}_{x}-\partial_{y 1} \hat{u}_{y}\right) \\
+A_{88} \rho\left(\partial_{x 1} \hat{u}_{y}+\partial_{y 1} \hat{u}_{x}\right)+\mathbf{B}_{8} \cdot \nabla_{1} \rho+\mathbf{C}_{8} \cdot \nabla_{1} \eta
\end{array}\right] .
$$

With the help of Eq. (A7), the nonzero elements $R_{81}, R_{87}, R_{88}$, $\mathbf{T}_{8}$, and $\mathbf{X}_{8}$ can be determined from Eq. (A13) as follows:

$$
\begin{aligned}
\boldsymbol{\Psi}\left[\begin{array}{l}
\left(2-s_{e}\right) R_{81} \\
\left(2-s_{p}\right) R_{87} \\
\left(2-s_{p}\right) R_{88}
\end{array}\right]=2\left(2-s_{p}\right)\left[\begin{array}{l}
A_{81} \\
A_{87} \\
A_{88}
\end{array}\right], \\
\mathbf{T}_{8}=-\frac{2-s_{p}}{2} \mathbf{B}_{8}+\frac{2-s_{e}}{4} R_{81} \mathbf{B}_{1}+\frac{2-s_{p}}{4} R_{87} \mathbf{B}_{7} \\
+\frac{2-s_{p}}{4} R_{88} \mathbf{B}_{8},
\end{aligned}
$$

$$
\begin{aligned}
\mathbf{X}_{8}= & -\frac{2-s_{p}}{2} \mathbf{C}_{8}+\frac{2-s_{e}}{4} R_{81} \mathbf{C}_{1}+\frac{2-s_{p}}{4} R_{87} \mathbf{C}_{7} \\
& +\frac{2-s_{p}}{4} R_{88} \mathbf{C}_{8}
\end{aligned}
$$

Compared with the original work by Huang et al. [17], the present improved scheme does not require the approximations $\mathbf{R} \sim \mathcal{O}\left(\mathrm{Ma}_{\text {lattice }}^{2}\right), \mathbf{T} \sim \mathcal{O}\left(\mathrm{Ma}_{\text {lattice }}^{3}\right)$, and $\mathbf{X} \sim$ $O\left(\mathrm{Ma}_{\text {lattice }}^{3}\right)$.
[1] Y. Wang, C. Shu, T. G. Wang, and P. V. Y. Alvarado, Phys Fluids 31, 103603 (2019).

[2] J. Ma, Z. Wang, J. Young, J. C. S. Lai, Y. Sui, and F.-B. Tian, J. Comput. Phys. 415, 109487 (2020).

[3] A. Eshghinejadfard, L. Daróczy, G. Janiga, and D. Thévenin, Int. J. Heat Fluid Flow 62, 93 (2016).

[4] X. Meng and Z. Guo, Int. J. Heat Mass Transfer 100, 767 (2016).

[5] D. L. Albernaz, M. Do-Quang, J. C. Hermanson, and G. Amberg, J. Fluid Mech. 820, 61 (2017).

[6] A. M. Moqaddam, S. S. Chikatamarla, and I. V. Karlin, Phys. Fluids 28, 022106 (2016).

[7] R. Huang, H. Wu, and N. A. Adams, Phys. Rev. E 100, 043306 (2019).

[8] G. Házi and A. Márkus, Int. J. Heat Mass Transfer 52, 1472 (2009).

[9] R. Huang and H. Wu, J. Comput. Phys. 277, 305 (2014).

[10] R. Huang and H. Wu, J. Comput. Phys. 315, 65 (2016).

[11] F. J. Alexander, S. Chen, and J. D. Sterling, Phys. Rev. E 47, R2249 (1993).

[12] L. Zheng, B. Shi, and Z. Guo, Phys. Rev. E 78, 026705 (2008).

[13] C. Coreixas, G. Wissocq, G. Puigt, J. F. Boussuge, and P. Sagaut, Phys. Rev. E 96, 033306 (2017).

[14] K. K. Mattila, P. C. Philippi, and L. A. Hegele, Phys. Fluids 29, 046103 (2017).

[15] R. Huang and H. Wu, Phys. Rev. E 89, 043303 (2014).

[16] M. H. Saadat, F. Bösch, and I. V. Karlin, Phys. Rev. E 99, 013306 (2019).

[17] R. Huang, H. Wu, and N. A. Adams, J. Comput. Phys. 392, 227 (2019).

[18] P. Lallemand and L.-S. Luo, Phys. Rev. E 68, 036706 (2003).

[19] Y.-L. Feng, S.-L. Guo, W.-Q. Tao, and P. Sagaut, Int. J. Heat Mass Transfer 125, 1379 (2018).

[20] D. L. Albernaz, M. Do-Quang, J. C. Hermanson, and G. Amberg, Phys. Fluids 28, 125105 (2016).
[21] X. Shan, Phys. Rev. E 55, 2780 (1997).

[22] Z. Guo, B. Shi, and C. Zheng, Int. J. Numer. Methods Fluids 39, 325 (2002).

[23] D. Contrino, P. Lallemand, P. Asinari, and L.-S. Luo, J. Comput. Phys. 275, 257 (2014).

[24] X. He, S. Chen, and G. D. Doolen, J. Comput. Phys. 146, 282 (1998).

[25] Z. Guo, C. Zheng, B. Shi, and T. S. Zhao, Phys. Rev. E 75, 036704 (2007).

[26] I. V. Karlin, D. Sichau, and S. S. Chikatamarla, Phys. Rev. E 88, 063310 (2013).

[27] Q. Li, K. H. Luo, Y. L. He, Y. J. Gao, and W. Q. Tao, Phys. Rev. E 85, 016710 (2012).

[28] Y. Feng, P. Sagaut, and W. Tao, J. Comput. Phys. 303, 514 (2015).

[29] N. I. Prasianakis and I. V. Karlin, Phys. Rev. E 76, 016702 (2007).

[30] L. Fei and K. H. Luo, Int. J. Therm. Sci. 132, 368 (2018).

[31] Y. H. Qian, D. d'Humières, and P. Lallemand, Europhys. Lett. 17, 479 (1992).

[32] R. Huang and H. Wu, J. Comput. Phys. 294, 346 (2015).

[33] P. Lallemand and L.-S. Luo, Phys. Rev. E 61, 6546 (2000).

[34] R. Huang, H. Wu, and N. A. Adams, Phys. Rev. E 99, 023303 (2019).

[35] R. Huang and H. Wu, J. Comput. Phys. 274, 50 (2014).

[36] R. Huang, H. Wu, and N. A. Adams, Phys. Rev. E 97, 053308 (2018).

[37] P. J. Dellar, J. Comput. Phys. 259, 270 (2014).

[38] Z. Chai and T. S. Zhao, Phys. Rev. E 90, 013305 (2014).

[39] T. Krüger, H. Kusumaatmaja, A. Kuzmin, O. Shardt, G. Silva, and E. M. Viggen, The Lattice Boltzmann Method: Principles and Practice (Springer, Cham, 2017).

[40] N. F. Carnahan and K. E. Starling, J. Chem. Phys. 51, 635 (1969). 
[41] H. W. Liepmann and A. Roshko, Elements of Gasdynamics (Wiley, New York, 1957).

[42] Y. A. Çengel and M. A. Boles, Thermodynamics: An Engineering Approach, 4th ed. (McGraw-Hill, Singapore, 2002).

[43] K. Nitsche and J. Straub, in Proceedings of the Sixth European Symposium on Material Sciences under Microgravity Condi- tions, ESA Spec. Publ. Vol. 256 (ESA, Paris, France, 1987), pp. 109-116.

[44] G. Accary, P. Bontoux, and B. Zappoli, J. Fluid Mech. 619, 127 (2009).

[45] B. Zappoli, D. Bailly, Y. Garrabos, B. Le Neindre, P. Guenoun, and D. Beysens, Phys. Rev. A 41, 2264 (1990). 